A quantum method for thermal rate constant calculations from stationary phase approximation of the thermal flux-flux correlation function integral

Chiara Aieta and Michele Ceotto

Citation: The Journal of Chemical Physics 146, 214115 (2017); doi: 10.1063/1.4984099

View online: http://dx.doi.org/10.1063/1.4984099

View Table of Contents: http://aip.scitation.org/toc/jcp/146/21

Published by the American Institute of Physics

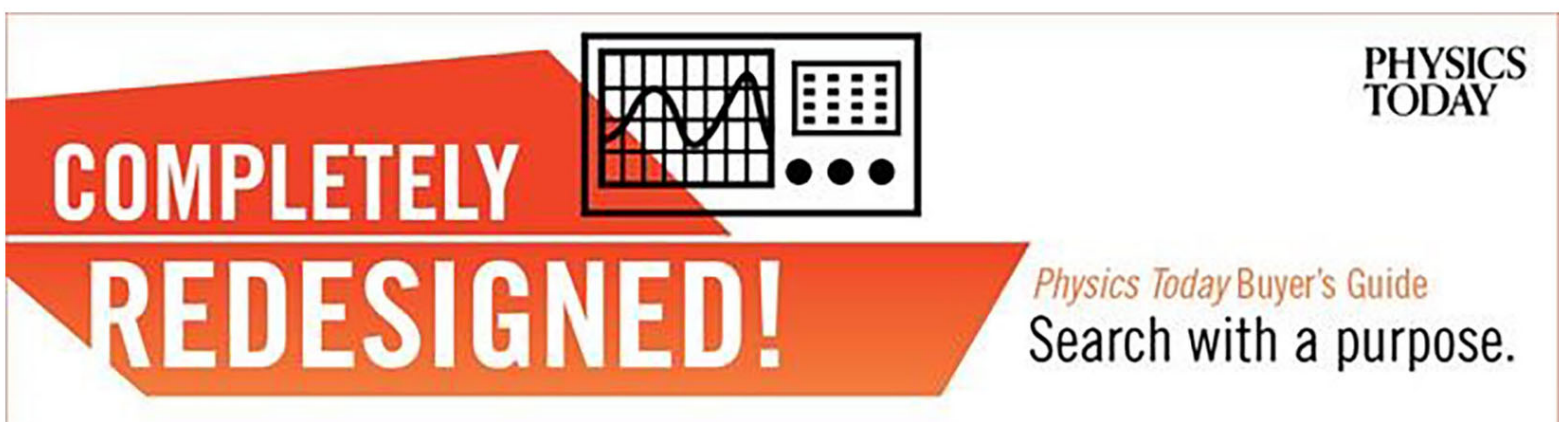




\title{
A quantum method for thermal rate constant calculations from stationary phase approximation of the thermal flux-flux correlation function integral
}

\author{
Chiara Aieta and Michele Ceotto \\ Dipartimento di Chimica, Università degli Studi di Milano, Via C. Golgi 19, 20133 Milano, Italy
}

(Received 14 February 2017; accepted 11 May 2017; published online 7 June 2017)

\begin{abstract}
This paper presents a quantum mechanical approximation to the calculation of thermal rate constants. The rate is derived from a suitable stationary phase approximation to the time integral of the thermal flux-flux correlation function. The goal is to obtain an expression that barely depends on the position of the flux operators, i.e., of the dividing surfaces, so that it can be applied also to complex systems by arbitrarily locating the dividing surfaces. The approach is tested on one and two dimensional systems where quantum effects are predominant over a wide range of temperatures. The results are quite accurate, i.e., within a few percent of the exact values for a reasonable range of dividing surface positions. Published by AIP Publishing. [http://dx.doi.org/10.1063/1.4984099]
\end{abstract}

\section{INTRODUCTION}

The calculation of the thermal reaction rate constant $\mathrm{k}(T)$ is a central problem in theoretical chemistry, and a quantum theory for its estimate is important because there are several physical processes including quantum nuclear tunneling and reflection where classical mechanics is not appropriate. To address this task, exact quantum expressions have been formulated, and these methods are well established nowadays. ${ }^{1,2}$ For example, a bimolecular reaction can be described in great detail by obtaining the S-matrix $S_{n p, n r}(\mathrm{E}, \mathrm{J})$ as a function of total energy E, and total angular momentum J. $S_{n p, n r}(\mathrm{E}, \mathrm{J})$ must be calculated for each open channel, i.e., for all reactive processes going from the quantum ro-vibrational state of the reactants (nr) to the product state (np), which are compatible with the $\mathrm{E}$ and $\mathrm{J}$ values. However, in the vast majority of chemical applications, the quantity of interest is $\mathrm{k}(T)$. In those cases, it is inefficient to calculate the full S-matrix and then sum up all state-to-state probabilities to get the full rate estimate. Instead, it is the more convenient "direct" and "correct" way introduced by Miller, where the rate $\mathrm{k}(T)$ is obtained by integrating over time the quantum flux-flux correlation $C_{f f}(t)$,

$$
k(T) Q_{r}(T)=\int_{0}^{\infty} C_{f f}(t) d t
$$

where $Q_{r}(T)$ is the reactant partition function. A possible quantum expression for $C_{f f}(t)$ has been proposed by Miller et al., ${ }^{3}$

$$
C_{f f}(t)=\operatorname{Tr}\left[\hat{F}_{1} e^{-\frac{\beta \hat{H}}{2}+\frac{i \hat{H} t}{\hbar}} \hat{F}_{2} e^{-\frac{\beta \hat{H}}{2}-\frac{i \hat{H} t}{\hbar}}\right],
$$

where the thermal part has been symmetrized. In Eq. (1), $\hat{F}_{1}$ and $\hat{F}_{2}$ are the quantum flux operators through two different dividing surfaces (DSs) defined in the coordinate space, where $\hat{F}_{i} \equiv \frac{d}{d t} \hat{h}_{i}$ and $\hat{h}_{i}$ is the Heavyside operator at the i-esime DS location, $\hat{H}$ is the quantum Hamiltonian operator, and $\beta$ $=1 / k_{B} \mathrm{~T}$ is the reciprocal of the temperature where $\mathrm{k}_{B}$ is the
Boltzmann constant. Equation (2) has been proven to minimize re-crossing contributions, ${ }^{4}$ which suggests that Eq. (2) is particularly suitable as a starting point for any approximation that would avoid the explicit time-integration, and it stresses the symmetry of the "complex time" forward and backward evolution operators. This method has been applied using Discrete Variable Representations (DVRs) for the calculations of thermal rate constants of several reactive systems ${ }^{5-9}$ Furthermore, by combining the multi-configurational time-dependent Hartree (MCTDH) approach and the flux-flux correlation function formalism, a direct calculation of accurate thermal rate constants was obtained for systems up to twelve degrees of freedom. ${ }^{10-12}$ However, the evaluation of the quantum mechanical trace in Eq. (2) and the time evolution of Eq. (1) remain computationally demanding in higher dimensional systems. For these reasons, approximate approaches are necessary when dealing with complex reactions. The semiclassical initial value representation (SC-IVR) and its approximations, such as the linearized SC-IVR (LSC-IVR), the forward-backward SC-IVR (FB-IVR), and the Van Vleck SC-IVR (VV-IVR), have also been applied for the calculation of the thermal rate, solving Eqs. (1) and (2) with phase space real-time classical trajectory integration. ${ }^{13-21}$

A well known approximate approach for the rate constant evaluation is the Transition State Theory (TST). TST is a zero-time classical approximation that relies on the direct dynamics approximation, ${ }^{22,23}$ where any re-crossings across the DS are neglected. Thus TST provides an upper bound to the exact classical rate constant and ignores any quantum effects, ${ }^{24}$ such as tunneling and corner cutting. Given these limitations, a quantum analog of TST is still under search. The main reason is that it is difficult to have a universal definition of Quantum Transition State Theory (QTST) because, opposite to classical TST, ${ }^{25,26}$ in QTST any local evaluation of the reactive flux is invalidated by the uncertainty principle. The main advantage of a QTST approach is that no real time evolution is requested. ${ }^{27-30}$ QTSTs are amenable to the 
imaginary time path integral formulation, ${ }^{31}$ thus avoiding to face the difficult sign problem, which hampers the application of other quantum ${ }^{6,32,33}$ or semiclassical methods explicitly including real-time evolution for the calculations of rate constants. ${ }^{13-16,18,19,21,34-54}$

The most straightforward inclusion of quantum effects in a TST formulation is to use the exact quantum partition functions for reactants and transition states. A more refined strategy is to employ semiclassical theories, which have already been successfully employed in many fields of theoretical chemistry. ${ }^{54-57}$ In kinetics, the main semiclassical theories are RiceRamsperger-Kassel-Marcus (RRKM), Semiclassical Transition State Theory (SCTST), ${ }^{27,58-60}$ and Semiclassical Instanton (SCI). ${ }^{27,61,62}$ If the real-time evolution is approximated with classical dynamics and the thermal part calculated by path integral techniques, the state of the art is represented by Ring Polymer Molecular Dynamics (RPMD), ${ }^{49,63,64}$ which can provide TST-like versions, ${ }^{65}$ and Centroid Molecular Dynamics (CMD) ${ }^{69-71}$

Among QTST methods, the one that is of particular interest for the present work is the Quantum Instanton (QI). ${ }^{45,69,70}$ QI is inspired from the semiclassical instanton theory ${ }^{27}$ but adopts a path integral treatment for the quantum Boltzmann statistics. QI has been proven to incorporate correctly all tunneling, corner-cutting, and quantum-fluctuation effects and has provided very satisfactory results for a variety of problems, ranging from gas phase reactions ${ }^{20,72-75}$ to chemical reactions in a polar solvent ${ }^{31}$ and from gas-surface reactions ${ }^{73,74}$ to isotopic effects. ${ }^{75-79}$ On one hand, the method has many appreciable features. First, it is very accurate, within $20 \%$, over a wide temperature range, from the deep tunneling regime up to the higher temperatures. Second, it makes no arbitrary assumptions about a specific reaction path or reaction coordinate. Finally, the only quantity which has to be evaluated is the Boltzmann operator (and its thermodynamic $\beta$ derivatives) that can be estimated by well known imaginary time path integral techniques. ${ }^{20,80}$ On the other hand, the QI approach presents a few drawbacks. First, it needs a fitted potential energy surface, which may be difficult to construct for complex or high-dimensional systems despite recent advances in the field ${ }^{81-91}$ Second, like any other QTST, QI does not reproduce recrossing effects, which are present in a quantum world both along the reactive and the non-reactive coordinates (i.e., the manifold space perpendicular to the DS).

In this paper, we introduce a quantum formulation for the calculation of the thermal rate constant with the main goal of reducing the strong DS dependency of previous QTST formulations. The method is obtained by the stationary phase approximating the time integral of the thermal flux-flux correlation function. The idea is pictorially described in Fig. 1, where a wave function is transmitted across a barrier. Differently from classical TST and some previous QTST approaches, we propose a two-DS approximation, where the DSs are arbitrarily located apart, i.e., on opposite sides of the barrier. The idea is to have the wave packet propagating from one DS to the barrier, tunneling through the barrier, and finally ending up to the products' side DS through an additional real time quantum propagation. The picture can be easily extended to

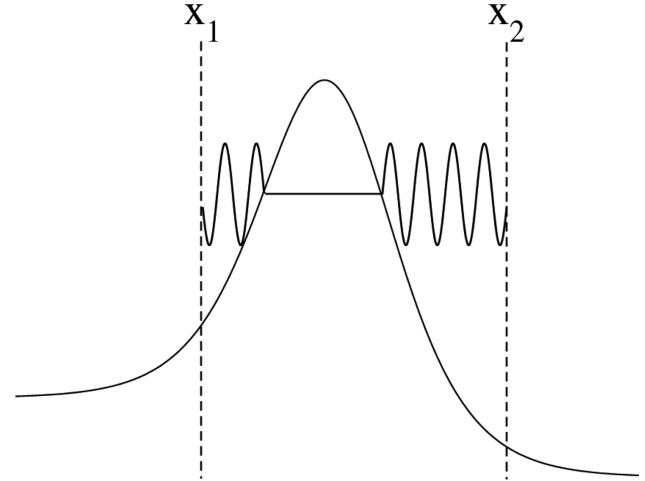

FIG. 1. Pictorial representation of the method presented here: the thermal rate constant is calculated at fixed DS positions by evaluating the quantum real and imaginary time propagator for a fixed amount of total time.

many dimensions by considering the wave packet propagating along any energy path. The amount of real versus imaginary time and the expression of the quantum propagator that follow this picture are not obtained by the product of three (two real and one imaginary time) propagators. Since all paths should be taken into account, this approach would end up in a real-time path integral calculation, which is numerically quite challenging. Instead, we will show below how the rate expression and the amount of real versus imaginary time can be found directly through a stationary phase approximation to the fluxflux correlation function time integral. Given this picture, any re-crossing effect is greatly reduced, since the two DSs are far apart, one on the reactants and the other one on the products' valley. In particular, to obtain the rate estimate, we will show that one needs only a single time evaluation of the flux-flux correlation function, which is the time $t_{s}$ at which a given quantity is stationary. When $\mathrm{t}_{s}=0$, one may obtain the QI formulation, a typical QTST approximation. When $\mathrm{t}_{s} \neq 0$, the estimate of the thermal rate is much less dependent on the DS position than other QTST formulations. This is a very important feature, since the best DS location is not trivial to find for complex systems.

The paper is organized as follows. In Sec. II, we introduce our stationary phase approximation to the thermal flux-flux correlation function integral. In Sec. II, we show how the approximation is related to the QI one, and we apply it to an analytically solvable problem, i.e., the free particle. In Sec. III, we test the method for the symmetric and asymmetric Eckart barriers and for the two collinear reactions $\mathrm{H}+\mathrm{H}_{2}$ and $\mathrm{D}+\mathrm{H}_{2}$. Finally, results are compared to some approximate methods for the calculation of thermal reaction rate constants and to exact values. In Sec. IV, we conclude and provide some perspective developments.

\section{THE METHOD}

\section{A. The approximate thermal rate expression}

This paragraph presents the main idea of this paper and introduces an approximate expression for the thermal rate constant. The derivation is done for the one dimensional case. However, the results can be easily generalized to many dimensions. 
We start from the exact quantum rate expression given by Eqs. (1) and (2). The value of the exact thermal rate constant $\mathrm{k}(T)$, which is the shaded area under the curves of Fig. 2, is independent from the position of the two DSs. However, the shape of the flux-flux correlation function significantly changes depending on the position of the DSs, as shown in Fig. 2. More specifically, when the two DSs are coincident, i.e., when $\hat{F}_{1}=\hat{F}_{2}=\hat{F}$, the profile of $C_{f f}(t)$ has its maximum at time $\mathrm{t}=0$. Once the two DSs are separated and the distance between them increased, the correlation function becomes double bell shaped with the maximum moving away from $\mathrm{t}=0$.

Once the DSs are far enough, the flux-flux correlation function value for $\mathrm{t}=0$ is $C_{f f}(0) \approx 0$. We now use the symmetry property of $C_{f f}(t)$, which is an even function of time, and extend the integration limit of Eq. (1) to $-\infty$ by taking half the integral

$$
k(T) Q_{r}(T)=\frac{1}{2} \int_{-\infty}^{+\infty} C_{f f}(t) d t .
$$

By multiplying and dividing the integrand by the positivedefinite term $\left|\left\langle x_{1}\left|e^{-\frac{\beta \hat{H}}{2}-\frac{i \hat{H} t}{\hbar}}\right| x_{2}\right\rangle\right|^{2}$ where $\mathrm{x}_{1}$ and $\mathrm{x}_{2}$ are the DS positions, we can restate the rate equation (3) in an equivalent form

$$
k(T) Q_{r}(T)=\frac{1}{2} \int_{-\infty}^{+\infty} \frac{C_{f f}(t)}{\left|\left\langle x_{1}\left|e^{-\frac{\beta \hat{H}}{2}-\frac{i \hat{H} t}{\hbar}}\right| x_{2}\right\rangle\right|^{2}} e^{\ln \left|\left\langle x_{1}\left|e^{-\frac{\beta \hat{H}}{2}-\frac{i \hat{H} t}{\hbar}}\right| x_{2}\right\rangle\right|^{2}} d t .
$$

We have numerically observed that depending on the DS location, there is a certain range where $C_{f f}(t) /\left|\left\langle x_{1}\left|e^{-\frac{\beta \hat{H}}{2}-\frac{i \hat{H} t}{\hbar}}\right| x_{2}\right\rangle\right|^{2}$

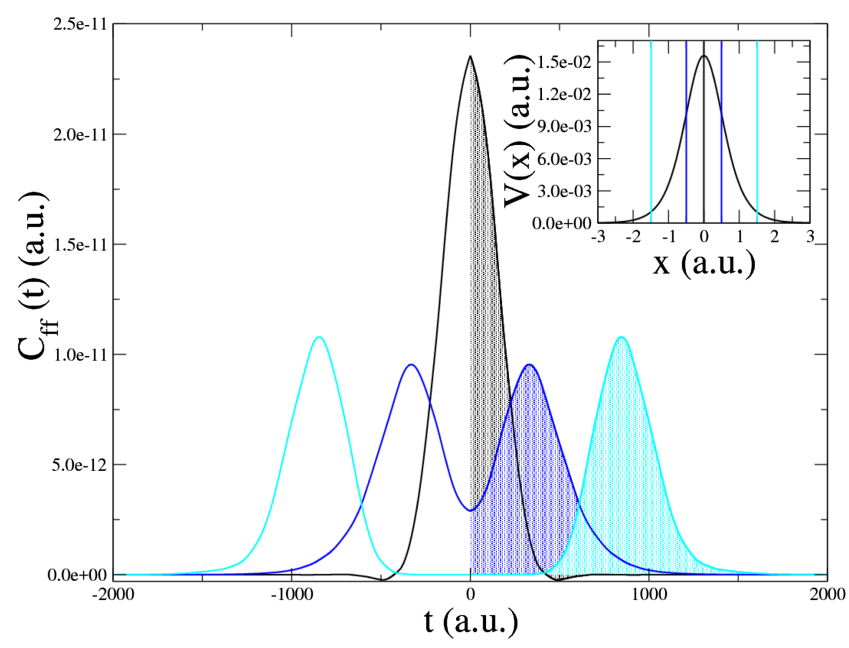

FIG. 2. Shape of the flux-flux correlation function at different DS locations for the Eckart barrier at $400 \mathrm{~K}$. The black curve is obtained when the two DSs are both located on the top of the barrier, as shown for the potential depicted in the inset. By increasing the distance between the two DSs, the shape of the flux-flux correlation function gradually changes as shown by the blue and the cyan curves. The shaded area represents the value of the rate constant which is independent from the position of the two DSs. These features are general and independent from the particular potential and temperature considered. is a slowly varying function of time and exp $\left[\ln \mid\left\langle x_{1}\right|\right.$ $\left.\left.e^{-\frac{\beta \hat{H}}{2}-\frac{i \hat{H} t}{\hbar}}\left|x_{2}\right\rangle\right|^{2}\right]$ is the fast varying part of the integrand. Under this condition, we can evaluate the time integral of Eq. (3) by the stationary phase approximation. The stationary phase prescription implies that

$$
\frac{d}{d t} \ln \left|\left\langle x_{1}\left|e^{-\frac{\beta \hat{H}}{2}-\frac{i \hat{H} t}{\hbar}}\right| x_{2}\right\rangle\right|_{t=t_{s}}^{2}=0
$$

where $t_{s}$ is the time when the phase is stationary. Equation (5) implies that

$$
\begin{gathered}
-\frac{i}{\hbar} \frac{\left\langle x_{1}\left|\hat{H} e^{-\frac{\beta \hat{H}}{2}-\frac{i \hat{H} t_{s}}{\hbar}}\right| x_{2}\right\rangle}{\left\langle x_{1}\left|e^{-\frac{\beta \hat{H}}{2}-\frac{i \hat{H} t_{s}}{\hbar}}\right| x_{2}\right\rangle}+\frac{i}{\hbar}\left(\frac{\left\langle x_{1}\left|\hat{H} e^{-\frac{\beta \hat{H}}{2}-\frac{i \hat{H} t_{s}}{\hbar}}\right| x_{2}\right\rangle}{\left\langle x_{1}\left|e^{-\frac{\beta \hat{H}}{2}-\frac{i \hat{H} t_{s}}{\hbar}}\right| x_{2}\right\rangle}\right)^{*} \\
=-\frac{i}{\hbar}\left[E\left(\beta, t_{s}\right)-E^{*}\left(\beta, t_{s}\right)\right]=\frac{2}{\hbar} \operatorname{Im}\left[E\left(\beta, t_{s}\right)\right]=0,
\end{gathered}
$$

where we have defined

$$
E(\beta, t)=\frac{\left\langle x_{1}\left|\hat{H} e^{-\frac{\beta \hat{H}}{2}-\frac{i \hat{H} t}{\hbar}}\right| x_{2}\right\rangle}{\left\langle x_{1}\left|e^{-\frac{\beta \hat{H}}{2}-\frac{i \hat{H} t}{\hbar}}\right| x_{2}\right\rangle} .
$$

$E(\beta, t)$ is an odd function of time and $\mathrm{t}_{s}=0$ is always a solution of Eq. (6). Therefore, independently from the system considered, there will be for all DS positions at least one solution $\left(\mathrm{t}_{s}=0\right)$, and if $\mathrm{t}_{s} \neq 0$ is an additional stationary time, also $-\mathrm{t}_{s}$ will be a stationary point. A typical plot of $\operatorname{Im}[E(\beta, t)]$ versus time is reported in Fig. 3. We can see that the DS position can be chosen such that $t_{\mathrm{s}}$ is smaller than the flux-flux correlation function decay time, i.e., $\hbar \beta .^{3}$

Now we expand the exponent in Eq. (4) in the Taylor series up to second order around each stationary time $t_{s}$ point

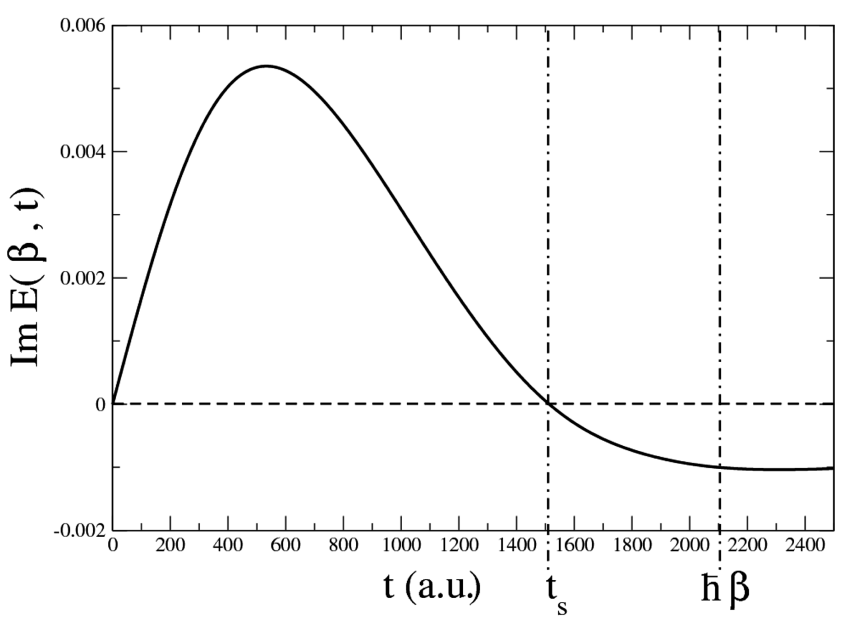

FIG. 3. Typical plot of $\operatorname{Im}[E(\beta, t)]$ for the Eckart barrier at $150 \mathrm{~K}$. The DSs are placed at 4.62 a.u. distance. The $C_{f f}(t)$ decay time $\hbar \beta$ is indicated by a vertical dashed-dotted line as well as the stationary time $\mathrm{t}_{\mathrm{s}}$. 


$$
\ln \left|\left\langle x_{1}\left|e^{-\frac{\beta \hat{H}}{2}-\frac{i \hat{H} t}{\hbar}}\right| x_{2}\right\rangle\right|^{2} \approx \ln \left|\left\langle x_{1}\left|e^{-\frac{\beta \hat{H}}{2}-\frac{i \hat{H} t_{s}}{\hbar}}\right| x_{2}\right\rangle\right|^{2}+\left.\left(t-t_{s}\right) \frac{d}{d t} \ln \left|\left\langle x_{1}\left|e^{-\frac{\beta \hat{H}}{2}-\frac{i \hat{H} t}{\hbar}}\right| x_{2}\right\rangle\right|^{2}\right|_{t=t_{s}}+\frac{\left(t-t_{s}\right)^{2}}{2} \frac{d^{2}}{d t^{2}} \ln \left|\left\langle x_{1}\left|e^{-\frac{\beta \hat{H}}{2}-\frac{i \hat{H} t}{\hbar}}\right| x_{2}\right\rangle\right|^{2} \mid
$$

By taking the second derivative

$$
\left.\frac{d^{2}}{d t^{2}} \ln \left|\left\langle x_{1}\left|e^{-\frac{\beta \hat{H}}{2}-\frac{i \hat{H} t}{\hbar}}\right| x_{2}\right\rangle\right|^{2}\right|_{t=t_{s}}=-\frac{2}{\hbar^{2}} \operatorname{Re} \Delta H^{2}\left(\beta, t_{s}\right)
$$

we evaluate the stationary-phase approximation,

$$
\begin{aligned}
k(T) Q_{r}(T) & \approx \sum_{t_{s}} \frac{1}{2} \int_{-\infty}^{+\infty} d t \frac{C_{f f}\left(t_{s}\right)}{\left|\left\langle x_{1}\left|e^{-\frac{\beta \hat{H}}{2}-\frac{i \hat{H} t_{s}}{\hbar}}\right| x_{2}\right\rangle\right|^{2}} e^{\left.\ln || x_{1}\left|e^{-\frac{\beta \hat{H}}{2}-\frac{i \hat{H} t_{s}}{\hbar}}\right| x_{2}\right\rangle\left.\right|^{2}+\frac{\left(t-t_{s}\right)^{2}}{2}\left(-\frac{2}{\hbar^{2}} \operatorname{Re} \Delta H^{2}\left(\beta, t_{s}\right)\right)} \\
& =\sum_{t_{s}} \frac{1}{2} \frac{C_{f f}\left(t_{s}\right)}{\left|\left\langle x_{1}\left|e^{-\frac{\beta \hat{H}}{2}-\frac{i \hat{H} t_{s}}{\hbar}}\right| x_{2}\right\rangle\right|^{2}} e^{\ln \left|\left\langle x_{1}\left|e^{-\frac{\beta \hat{H}}{2}-\frac{i \hat{H} t_{s}}{\hbar}}\right| x_{2}\right\rangle\right|^{2}} \int_{-\infty}^{+\infty} d t e^{-\frac{\left(t-t_{s}\right)^{2}}{\hbar^{2}} \operatorname{Re} \Delta H^{2}\left(\beta, t_{s}\right)},
\end{aligned}
$$

and after the Gaussian integration at each stationary point, we obtain

$$
k(T) Q_{r}(T) \approx \sum_{t_{s}} \frac{1}{2} \frac{\hbar \sqrt{\pi}}{\sqrt{\operatorname{Re} \Delta H^{2}\left(\beta, t_{s}\right)}} C_{f f}\left(t_{s}\right),
$$

where

$$
\Delta H^{2}\left(\beta, t_{s}\right)=\frac{\left\langle x_{1}\left|\hat{H}^{2} e^{-\frac{\beta \hat{H}}{2}-\frac{i \hat{H} t_{s}}{\hbar}}\right| x_{2}\right\rangle}{\left\langle x_{1}\left|e^{-\frac{\beta \hat{H}}{2}-\frac{i \hat{H} t_{s}}{\hbar}}\right| x_{2}\right\rangle}-\left(\frac{\left.\left\langle x_{1}\left|\hat{H} e^{-\frac{\beta \hat{H}}{2}-\frac{i \hat{H} t_{s}}{\hbar}}\right| x_{2}\right\rangle\right)^{2}}{\left\langle x_{1}\left|e^{-\frac{\beta \hat{H}}{2}-\frac{i \hat{H} t_{s}}{\hbar}}\right| x_{2}\right\rangle}\right)^{2}
$$

and $C_{f f}\left(t_{s}\right)$ is the flux-flux correlation function value at time $\mathrm{t}=\mathrm{t}_{s}$ defined as

$$
C_{f f}\left(t_{s}\right)=\operatorname{Tr}\left[\hat{F}_{1} e^{-\frac{\beta \hat{H}}{2}+\frac{i \hat{H} t_{s}}{\hbar}} \hat{F}_{2} e^{-\frac{\beta \hat{H}}{2}-\frac{i \hat{H} t_{s}}{\hbar}}\right] .
$$

From the symmetry considerations pointed out above, and considering that both the flux-flux correlation function and $\operatorname{Re} \Delta H^{2}\left(\beta, t_{s}\right)$ are even functions of time, we can restate Eq. (11) as the sum

$$
\begin{aligned}
k(T) Q_{r}(T) & \approx \frac{1}{2} \frac{\hbar \sqrt{\pi} C_{f f}\left(t_{s}=0\right)}{\sqrt{\operatorname{Re} \Delta H^{2}\left(\beta, t_{s}=0\right)}}+\frac{1}{2} \frac{\hbar \sqrt{\pi} C_{f f}\left(t_{s}\right)}{\sqrt{\operatorname{Re} \Delta H^{2}\left(\beta, t_{s}\right)}}+\frac{1}{2} \frac{\hbar \sqrt{\pi} C_{f f}\left(-t_{s}\right)}{\sqrt{\operatorname{Re} \Delta H^{2}\left(\beta,-t_{s}\right)}} \\
& =\frac{1}{2} \frac{\hbar \sqrt{\pi} C_{f f}\left(t_{s}=0\right)}{\sqrt{\operatorname{Re} \Delta H^{2}\left(\beta, t_{s}=0\right)}}+\frac{\hbar \sqrt{\pi} C_{f f}\left(t_{s}\right)}{\sqrt{\operatorname{Re} \Delta H^{2}\left(\beta, t_{s}\right)}},
\end{aligned}
$$

which includes a single value of $\mathrm{t}_{s} \neq 0$. Finally, recalling that we have chosen the two DSs sufficiently far apart that $C_{f f}(0)=0$, we obtain the central result of this paper

$$
k(T) Q_{r}(T) \approx \frac{\hbar \sqrt{\pi}}{\sqrt{\operatorname{Re} \Delta H^{2}\left(\beta, t_{s}\right)}} C_{f f}\left(t_{s}\right) .
$$

Following the work of Miller et al., ${ }^{72}$ Eq. (15) can be extended to multidimensional problems by taking

$$
\begin{aligned}
\Delta H^{2}\left(\beta, t_{s}\right) \approx\left\langle\Delta H^{2}\left(\beta, t_{s}\right)\right\rangle= & \frac{\int d \mathbf{Y}_{\mathbf{1}} \int d \mathbf{Y}_{\mathbf{2}}\left\langle x_{1} \mathbf{Y}_{\mathbf{1}}\left|\hat{H}^{2} e^{-\frac{\beta \hat{H}}{2}-\frac{i \hat{H} t_{s}}{\hbar}}\right| x_{2} \mathbf{Y}_{\mathbf{2}}\right\rangle\left\langle x_{1} \mathbf{Y}_{\mathbf{1}}\left|e^{-\frac{\beta \hat{H}}{2}-\frac{i \hat{H} t_{s}}{\hbar}}\right| x_{2} \mathbf{Y}_{\mathbf{2}}\right\rangle}{\int d \mathbf{Y}_{\mathbf{1}} \int d \mathbf{Y}_{\mathbf{2}}\left(\left\langle x_{1} \mathbf{Y}_{\mathbf{1}}\left|e^{-\frac{\beta \hat{H}}{2}-\frac{i \hat{H} t_{s}}{\hbar}}\right| x_{2} \mathbf{Y}_{\mathbf{2}}\right\rangle\right)^{2}} \\
& -\frac{\int d \mathbf{Y}_{\mathbf{1}} \int d \mathbf{Y}_{\mathbf{2}}\left(\left\langle x_{1} \mathbf{Y}_{\mathbf{1}}\left|\hat{H} e^{-\frac{\beta \hat{H}}{2}-\frac{i \hat{H} t_{s}}{\hbar}}\right| x_{2} \mathbf{Y}_{\mathbf{2}}\right\rangle\right)^{2}}{\int d \mathbf{Y}_{\mathbf{1}} \int d \mathbf{Y}_{\mathbf{2}}\left(\left\langle x_{1} \mathbf{Y}_{\mathbf{1}}\left|e^{-\frac{\beta \hat{H}}{2}-\frac{i \hat{H} t_{s}}{\hbar}}\right| x_{2} \mathbf{Y}_{\mathbf{2}}\right\rangle\right)^{2}}
\end{aligned}
$$

where the average is over the $\mathbf{Y}$ coordinates, i.e., the set of coordinates orthogonal to the reactive one at the TST location. This approximation is exact in the separable limit, and it is an extension to complex time of what was previously derived for purely imaginary time. $^{72}$

In summary, the procedure for the approximate evaluation of the thermal rate constant is first to fix the two DSs 
sufficiently far apart such that $C_{f f}(0)=0$. Then, find the zero of Eq. (6) and finally, evaluate Eq. (15) at the time $t_{s}$.

\section{B. An alternative derivation of the quantum instanton approximation}

In this paragraph, we show that the same procedure presented above can be employed to derive the QI expression, at least in its simplest one DS version. To prove this, we first choose the two DSs to be the same, i.e., $\mathrm{x}_{1}=\mathrm{x}_{2}=\mathrm{x}_{0}$,

$k(T) Q_{r}(T)=\frac{1}{2} \int_{-\infty}^{+\infty} d t \frac{C_{f f}(t)}{\left|\left\langle x_{0}\left|e^{-\frac{\beta \hat{H}}{2}-\frac{i \hat{H} t}{\hbar}}\right| x_{0}\right\rangle\right|^{2}} e^{\ln \left|\left\langle x_{0}\left|e^{-\frac{\beta \hat{H}}{2}-\frac{i \hat{H} t}{\hbar}}\right| x_{0}\right\rangle\right|^{2} .}$

We now remember that the QI approximation is a zero-time approximation, i.e., a proper QTST approximation, differently from Eq. (15). To retrieve its expression, one could naively impose $t=0$ in Eq. (17), but in this way the integral is too approximated. Hence, we expand the exponent in Eq. (17) up to the second order around the QI stationary time $\mathrm{t}=0$,

$$
\begin{aligned}
\ln \left|\left\langle x_{0}\left|e^{-\frac{\beta \hat{H}}{2}-\frac{i \hat{H} t}{\hbar}}\right| x_{0}\right\rangle\right|^{2} \simeq & \ln \left|\left\langle x_{0}\left|e^{-\frac{\beta \hat{H}}{2}}\right| x_{0}\right\rangle\right|^{2} \\
& +\left.t \frac{d}{d t} \ln \left|\left\langle x_{0}\left|e^{-\frac{\beta \hat{H}}{2}-\frac{i \hat{H} t}{\hbar}}\right| x_{0}\right\rangle\right|^{2}\right|_{t=0} . \\
& +\left.\frac{t^{2}}{2} \frac{d^{2}}{d t^{2}} \ln \left|\left\langle x_{0}\left|e^{-\frac{\beta \hat{H}}{2}-\frac{i \hat{H} t}{\hbar}}\right| x_{0}\right\rangle\right|^{2}\right|_{t=0}
\end{aligned}
$$

Then, we observe that the stationary condition is always satisfied since the 1.h.s. of Eq. (18) is an even function of time and the first order term vanishes for any choice of $x_{0}$. The second derivative is given by Eq. (9), and we obtain

$$
\begin{aligned}
k(T) Q_{r}(T)_{Q I}^{\text {approx }} & =\frac{1}{2} \int_{-\infty}^{+\infty} d t \frac{C_{f f}(0)}{\left|\left\langle x_{0}\left|e^{-\frac{\beta \hat{H}}{2}}\right| x_{0}\right\rangle\right|^{2}} e^{\ln \left|\left\langle x_{0}\left|e^{-\frac{\beta \hat{H}}{2}}\right| x_{0}\right\rangle\right|^{2}+\frac{t^{2}}{2}\left(-\frac{2}{\hbar^{2}} \operatorname{Re} \Delta H^{2}(\beta, 0)\right)} \\
& =\frac{1}{2} \frac{C_{f f}(0)}{\left|\left\langle x_{0}\left|e^{-\frac{\beta \hat{H}}{2}}\right| x_{0}\right\rangle\right|^{2}} e^{\ln \left|\left\langle x_{0}\left|e^{-\frac{\beta \hat{H}}{2}}\right| x_{0}\right\rangle\right|^{2}} \int_{-\infty}^{+\infty} d t e^{-\frac{t^{2}}{\hbar^{2}} \operatorname{Re} \Delta H^{2}(\beta, 0)} .
\end{aligned}
$$

We now perform the Gaussian integral and obtain the familiar QI expression ${ }^{69}$

$$
k(T) Q_{r}(T)_{Q I}^{\text {approx }}=\frac{1}{2} C_{f f}(0) \frac{\hbar \sqrt{\pi}}{\sqrt{\operatorname{Re} \Delta H^{2}(\beta, 0)}},
$$

where

$$
\Delta H^{2}(\beta, 0)=\frac{\left\langle x_{0}\left|\hat{H}^{2} e^{-\frac{\beta \hat{H}}{2}}\right| x_{0}\right\rangle}{\left\langle x_{0}\left|e^{-\frac{\beta \hat{H}}{2}}\right| x_{0}\right\rangle}-\left(\frac{\left\langle x_{0}\left|\hat{H} e^{-\frac{\beta \hat{H}}{2}}\right| x_{0}\right\rangle}{\left\langle x_{0}\left|e^{-\frac{\beta \hat{H}}{2}}\right| x_{0}\right\rangle}\right)^{2} .
$$

The QI rate in Eq. (20) can be applied to any $x_{0}$ dividing surface position. However, this single stationary time formulation is more accurate when the zeroth order term $\ln \left|\left\langle x_{0}\left|e^{-\frac{\beta \hat{H}}{2}}\right| x_{0}\right\rangle\right|^{2}$ is maximized with respect to $x_{0}$, i.e., $\partial\left\langle x_{0}\left|e^{-\frac{\beta \hat{H}}{2}}\right| x_{0}\right\rangle / \partial x_{0}=0$, which is the original QI dividing surface requirement. ${ }^{69}$

\section{An analytical case: The free particle}

In order to investigate the general high temperature limit of Eq. (15), we look at the free particle case. We start from the matrix elements

$$
\left\langle x_{1}\left|e^{-\left(\frac{\beta}{2}+\frac{i t}{\hbar}\right) \hat{H}}\right| x_{2}\right\rangle=\sqrt{\frac{m}{2 \pi \hbar^{2}\left(\frac{\beta}{2}+\frac{i t}{\hbar}\right)}} e^{-\frac{m\left(x_{2}-x_{1}\right)^{2}}{2 \hbar^{2}\left(\frac{\beta}{2}+\frac{i t}{\hbar}\right)}}
$$

and the squared modulus of the propagator

$$
\left|\left\langle x_{1}\left|e^{-\left(\frac{\beta}{2}+\frac{i t}{\hbar}\right) \hat{H}}\right| x_{2}\right\rangle\right|^{2}=\frac{m}{2 \pi \hbar \sqrt{t^{2}+\left(\frac{\beta \hbar}{2}\right)^{2}}} e^{-\frac{m\left(x_{2}-x_{1}\right)^{2} \beta}{2\left[t^{2}+\left(\frac{\beta \hbar}{2}\right)^{2}\right]}} .
$$

For the free particle and considering two distinct DSs, the flux-flux correlation function is

$$
\begin{aligned}
C_{f f}(t)_{f p}= & \frac{k_{B} T}{h} \frac{(\hbar \beta / 2)^{2}}{\left[t^{2}+\left(\frac{\beta \hbar}{2}\right)^{2}\right]^{3 / 2}}\left\{1+\frac{m \beta}{(\hbar \beta / 2)^{2}} \frac{\left(x_{2}-x_{1}\right) t^{2}}{\left[t^{2}+\left(\frac{\beta \hbar}{2}\right)^{2}\right]}\right\} \\
& \times \exp \left\{-\frac{m\left(x_{2}-x_{1}\right)^{2} \beta}{2\left[t^{2}+\left(\frac{\beta \hbar}{2}\right)^{2}\right]}\right\},
\end{aligned}
$$

as demonstrated at the end of Appendix A.

From Eq. (23), the stationary phase condition (5) is satisfied whenever the time variable $t$ is such that

$$
t\left\{\frac{m\left(x_{2}-x_{1}\right)^{2} \beta}{\left[t^{2}+\left(\frac{\beta \hbar}{2}\right)^{2}\right]^{2}}-\frac{1}{t^{2}+\left(\frac{\beta \hbar}{2}\right)^{2}}\right\}=0,
$$

or besides the solution $t_{s}=0$, when

$$
t_{s}= \pm \sqrt{m\left(x_{2}-x_{1}\right)^{2} \beta-\left(\frac{\beta \hbar}{2}\right)^{2}}
$$

Real time condition for $t_{s}$ implies that $\mathrm{m}\left(x_{2}-x_{1}\right)^{2} \beta>(\hbar \beta / 2)^{2}$. This inequality provides a lower bound for the minimum distance between the DSs at temperature $\beta$, according to the least uncertainty principle. More specifically, the above inequality can be written as

$$
m\left(x_{2}-x_{1}\right)^{2} / \beta=\Delta x^{2} m k_{B} T=\Delta x^{2} m^{2} v^{2}=\Delta x^{2} \Delta p^{2}>\left(\frac{\hbar}{2}\right)^{2},
$$


where the equipartition theorem $k_{B} \mathrm{~T} / 2=\mathrm{m} v^{2} / 2$ has been employed. The solution $t_{s}=0$ does not contribute to the approximate rate. In fact, the flux-flux correlation function given in Eq. (24) evaluated at this time gives

$$
C_{f f}(0)_{f p}=\frac{1}{\pi \hbar^{2} \beta^{2}} e^{-\frac{2 m\left(x_{2}-x_{1}\right)^{2}}{\beta \hbar^{2}}},
$$

and its value vanishes when taking the two DSs sufficiently distant from each other.

After evaluating

$$
\left.\left|\left\langle x_{1}\left|e^{-\left(\frac{\beta}{2}+\frac{i t}{\hbar}\right) \hat{H}}\right| x_{2}\right\rangle\right|^{2}\right|_{t=t_{s}}=\frac{m}{2 \pi \hbar \sqrt{m\left(x_{2}-x_{1}\right)^{2} \beta}} e^{-\frac{1}{2}},
$$

we obtain the ratio and evaluate it at $\mathrm{t}=t_{s}$,

$$
\left.\frac{C_{f f}(t)_{f p}}{\left|\left\langle x_{1}\left|e^{-\left(\frac{\beta}{2}+\frac{i t}{\hbar}\right) \hat{H}}\right| x_{2}\right\rangle\right|^{2}}\right|_{t=t_{s}}=\frac{1}{m \beta}
$$

By evaluating the second derivative of the phase, i.e., the first derivative of the stationary condition (25) at $t=t_{s}$, we obtain

$$
\begin{aligned}
\frac{d^{2}}{d t^{2}} \ln & \left(\left|\left\langle x_{1}\left|e^{-\left(\frac{\beta}{2}+\frac{i t}{\hbar}\right) \hat{H}}\right| x_{2}\right\rangle\right|^{2}\right) \mid \\
& =\frac{2}{m^{2}\left(x_{2}-x_{1}\right)^{4} \beta^{2}}\left[\left(\frac{\hbar \beta}{2}\right)^{2}-m\left(x_{2}-x_{1}\right)^{2} \beta\right]
\end{aligned}
$$

Finally, after the Gaussian integration, we get to the approximate free particle rate

$$
\begin{aligned}
Q_{r}(T) k(T)_{f p} \approx & \frac{1}{m \beta} \frac{m e^{-1 / 2}}{2 \pi \hbar \sqrt{m\left(x_{2}-x_{1}\right)^{2} \beta}} \sqrt{\pi} \\
& \times\left[-\frac{1}{2}\left[\frac{2\left(\left(\frac{\hbar \beta}{2}\right)^{2}-m\left(x_{2}-x_{1}\right)^{2} \beta\right)}{m^{2}\left(x_{2}-x_{1}\right)^{4} \beta^{2}}\right)\right]^{-1 / 2} \\
= & \frac{k_{B} T}{h} \sqrt{\frac{\pi}{e}} \sqrt{\frac{m\left(x_{2}-x_{1}\right)^{2} \beta}{m\left(x_{2}-x_{1}\right)^{2} \beta-\left(\frac{\beta \hbar}{2}\right)^{2}}} \\
& =\frac{k_{B} T}{h} \sqrt{\frac{\pi}{e}} \sqrt{1+\left(\frac{\hbar \beta}{2 t_{s}}\right)^{2}} .
\end{aligned}
$$

The free particle expression of Eq. (32) is quite accurate at high temperatures, regardless of the actual position of the two DSs. In fact, for small $\beta, Q_{r}(T) k(T)_{f p} \approx\left(k_{B} T / h\right) \sqrt{\pi / e}$ which is quite a good approximation of the exact $k_{B} T / h$ rate, since $\sqrt{\pi / e}=1.075$, and to be compared with the QI free particle limit equals to $\left(k_{B} T / h\right) \sqrt{\pi / 2} \cdot{ }^{69}$ At a low temperature, the accuracy depends on the position of the DSs. However, fixing the DSs such that $\mathrm{t}_{s} \sim \hbar \beta$, we obtain again a better accuracy than the QI one.

\section{RESULTS AND DISCUSSION}

\section{A. Results for the 1D symmetric and asymmetric Eckart barrier}

The one dimensional Eckart barrier problem is a standard test for approximate rate theories. The Eckart symmetric potential is

$$
V(x)=V_{0} \operatorname{sech}^{2}(\mathrm{a} x),
$$

and in this paper, the parameters are chosen to approximately model the $\mathrm{H}+\mathrm{H}_{2}$ minimum energy path, i.e., $V_{0}=0.425 \mathrm{eV}$, $\mathrm{a}=1.36$ a.u., and $\mathrm{m}=1060$ a.u. Another useful one dimensional potential is the Eckart asymmetric potential, which can be regarded as a model for a heteroatomic bimolecular reaction

$$
V(x)=\frac{V_{0}(1-\alpha)}{1+e^{-2 a x}}+\frac{V_{0}(1+\sqrt{\alpha})^{2}}{4 \cosh ^{2}(a x)} .
$$

In our tests, we set the parameters in Eq. (34) as $V_{0}=0.01562 \mathrm{eV}, \mathrm{a}=1.3624$ a.u., $\alpha=1.25$, and $\mathrm{m}=1060$ a.u. The potentials of Eqs. (33) and (34) are reported in Fig. 4. All the matrix elements needed to calculate Eq. (15) have been evaluated by diagonalizing the Hamiltonian in a DVR representation (sinc-DVR). ${ }^{95}$ For both potential profiles, we have obtained the estimates of the thermal rate constant at several positions of the two DSs (see Tables I and II) after evaluating the stationary time $t_{s}$ as shown in Fig. 3. Both for the symmetric and asymmetric potentials, we intentionally set the surfaces symmetrically with respect to the top of the barrier. We find that the results are just a few percentage away from the exact ones that we calculated by DVR, independently from the position of the two DSs

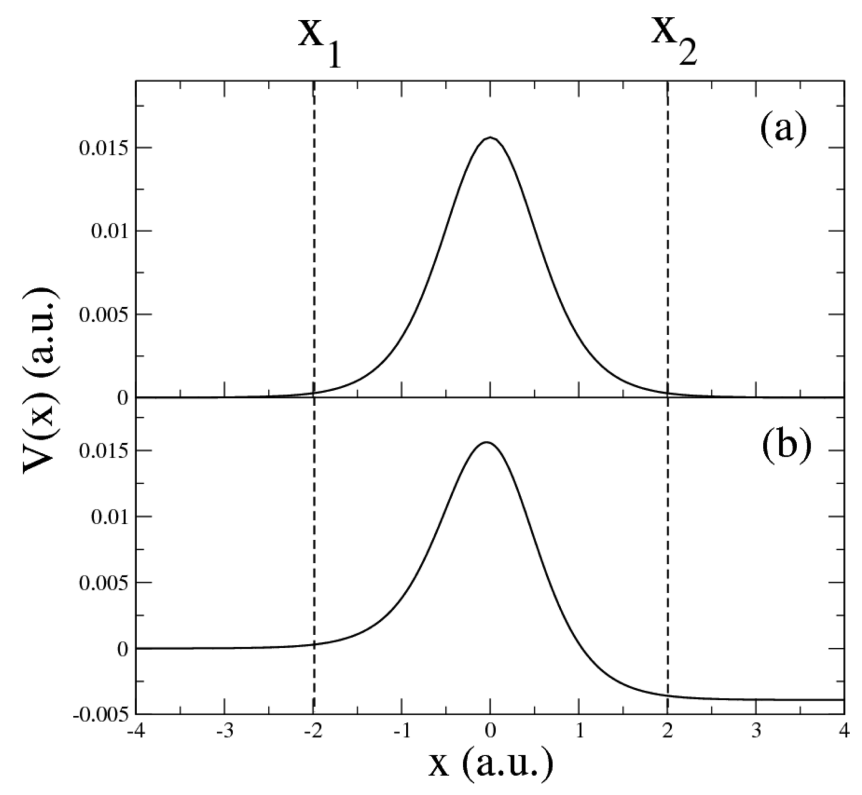

FIG. 4. The symmetric (a) and asymmetric (b) Eckart barrier potentials. The two dashed vertical lines located at $x_{1}$ and $x_{2}$ represent possible positions of the DSs. 
TABLE I. Percentage error $\left[\%\right.$ Err $\left.=100 \times\left(k^{\text {approx }}-k^{\text {exact }}\right) / k^{\text {exact }}\right]$ of the calculated rate constant with respect to the exact rate for the $1 \mathrm{D}$ symmetric Eckart potential of Eq. (33) at three temperatures $(150 \mathrm{~K}, 300 \mathrm{~K}$, and $1000 \mathrm{~K})$. $\mathrm{x}_{1}$ and $\mathrm{x}_{2}$ are the positions of the two DSs with respect to the top of the barrier.

\begin{tabular}{lcrrr}
\hline \hline & & \multicolumn{3}{c}{$\mathrm{T}(\mathrm{K})$} \\
\cline { 3 - 5 } $\mathrm{x}_{1}$ (a.u.) & $\mathrm{x}_{2}$ (a.u.) & 150 & 300 & 1000 \\
\hline-1.47 & 1.47 & $12.7 \%$ & $0.8 \%$ & $-3.9 \%$ \\
-1.75 & 1.75 & $7.0 \%$ & $2.5 \%$ & $-3.3 \%$ \\
-2.03 & 2.03 & $4.6 \%$ & $3.4 \%$ & $-2.8 \%$ \\
-2.31 & 2.31 & $0.9 \%$ & $2.5 \%$ & $-3.2 \%$ \\
-2.59 & 2.59 & $4.4 \%$ & $3.4 \%$ & $-1.8 \%$ \\
-2.87 & 2.87 & $2.0 \%$ & $1.3 \%$ & $-2.4 \%$ \\
-3.15 & 3.15 & $5.0 \%$ & $2.0 \%$ & $-1.2 \%$ \\
-3.43 & 3.43 & $1.4 \%$ & $-0.5 \%$ & $-1.9 \%$ \\
\hline \hline
\end{tabular}

TABLE II. The same as in Table I but for the asymmetric Eckart potential of Eq. (34).

\begin{tabular}{lcrcc}
\hline \hline & & \multicolumn{3}{c}{$\mathrm{T}(\mathrm{K})$} \\
\cline { 3 - 5 } $\mathrm{x}_{1}$ (a.u.) & $\mathrm{x}_{2}$ (a.u.) & 150 & 300 & 1000 \\
\hline-1.77 & 1.77 & $3.5 \%$ & $2.2 \%$ & $-5.2 \%$ \\
-2.30 & 2.30 & $-7.8 \%$ & $2.4 \%$ & $-4.2 \%$ \\
-2.83 & 2.83 & $-6.0 \%$ & $0.9 \%$ & $-3.4 \%$ \\
-3.37 & 3.37 & $-3.8 \%$ & $-1.5 \%$ & $-3.0 \%$ \\
-3.90 & 3.90 & $-3.6 \%$ & $-4.1 \%$ & $-2.5 \%$ \\
-4.43 & 4.43 & $-3.4 \%$ & $-6.8 \%$ & $-2.3 \%$ \\
-4.97 & 4.97 & $-3.3 \%$ & $-9.3 \%$ & $-2.1 \%$ \\
\hline \hline
\end{tabular}

and provided they are sufficiently far from each other. It is surprising that a symmetric DS disposition gives accurate results even for the asymmetric potential, and comparable in accuracy to those obtained for the symmetric barrier. Furthermore, the asymmetric potential results can be improved by choosing the DS positions in an asymmetric fashion, as shown in Table III.

To better appreciate the accuracy of the present approximation [Eq. (15)], we compare the results of Table I with other approximate approaches for the calculation of the thermal rate constants, as shown in Table IV. The present approach can predict very accurate rate constants (with errors below 13\%). In contrast, the RPMD, ${ }^{49}$ the Linearized Semiclassical Initial Value Representation (LSC-IVR), ${ }^{17}$ and the Semiclassical Van Vleck Initial Value Representation (SC-VV-IVR) ${ }^{17}$ methods
TABLE III. Percentage error $\left[\% \operatorname{Err}=100 \times\left(k^{\text {approx }}-k^{\text {exact }}\right) / k^{\text {exact }}\right]$ of the calculated rate constant with respect to the exact rate for different choices of the DS positions at $300 \mathrm{~K}$ in the asymmetric Eckart barrier case. Results are reported as a function of the position $\mathrm{x}_{2}$ of the right DS and for different settings of the left DS $\mathrm{x}_{1}$.

\begin{tabular}{lccc}
\hline \hline & \multicolumn{3}{c}{$\mathrm{x}_{1}$ (a.u.) } \\
\cline { 2 - 4 } $\mathrm{x}_{2}$ (a.u.) & -2.03 & -2.50 & -3.10 \\
\hline 2.03 & $2.6 \%$ & $2.4 \%$ & $1.4 \%$ \\
2.50 & $2.2 \%$ & $1.8 \%$ & $0.6 \%$ \\
3.10 & $2.0 \%$ & $1.2 \%$ & $-0.2 \%$ \\
3.63 & $1.6 \%$ & $0.4 \%$ & $-1.1 \%$ \\
4.17 & $1.2 \%$ & $-0.3 \%$ & $-1.8 \%$ \\
\hline \hline
\end{tabular}

cannot retain a high accuracy for the rate estimates in the deep tunneling regime for this one dimensional case. QI is very precise at low temperatures but looses accuracy in the high temperature limit. ${ }^{69}$ To correct this deviation, Miller et al. introduced an ad hoc free particle correction reported in brackets in Table IV. Also, a refined QI approach that relies on the Higher Derivatives of the flux-flux correlation function (HD-QI) is better performing over the entire temperature range, but the calculation of higher flux-flux correlation function derivatives can be quite demanding and the approach difficult to be applied to more complex systems. ${ }^{45}$ Remarkably, the new approximation of Eq. (15) is stable over a wide temperature range, from the deep tunneling regime up to higher temperatures without any ad hoc correction. Furthermore, our new approach provides a reliable estimate compared to preexisting methods even if the positions of the DSs have not been optimized.

\section{B. Application to the $\mathrm{H}+\mathrm{H}_{2}$ and $\mathrm{D}+\mathrm{H}_{2}$ reactions}

A severe and common multidimensional test for quantum transition state theories is without any doubt the collinear $\mathrm{H}+\mathrm{H}_{2}$ reaction, where the amount of tunneling and cornercutting for the rate calculation is prominent. Theories based on the vibrational adiabatic separation fail to correctly predict the rate for this simple bidimensional system because of the rapid change of the curvature for the potential energy surface around the saddle point. ${ }^{69}$ For these reasons, we tested Eq. (15) on the collinear $\mathrm{H}+\mathrm{H}_{2}$ reaction. To show that we do not take advantages from any symmetry property of the potential, we apply our rate approximation also to the collinear $\mathrm{D}+\mathrm{H}_{2}$ reaction. We have employed the Liu-Siegbahn-Truhlar-Horowitz (LSTH) potential energy surface. ${ }^{97,98}$ The matrix elements of

TABLE IV. Comparison of the percentage error $\left[\% \operatorname{Err}=100 \times\left(k^{\text {approx }}-k^{\text {exact }}\right) / k^{\text {exact }}\right]$ of the thermal rate constant with respect to the exact value for the symmetric Eckart barrier obtained with different approximate approaches. Values deduced graphically from the cited paper are indicated by the symbol $\sim$. The percentage error reported for the present method is the worst estimate obtained in Table I. Free particle corrected QI results in brackets.

\begin{tabular}{lcccccc}
\hline \hline $\mathrm{T}(\mathrm{K})$ & LSC-IVR $^{17}$ & SC-VV-IVR $^{17}$ & RPMD $^{49}$ & QI $^{72}$ & HD-QI $^{45}$ & Equation (15) \\
\hline 150 & $\sim-62$ & $\sim-33$ & $\ldots$ & $+1.7(-3.2)$ & $\sim+2$ & +12.7 \\
200 & $\sim-37$ & $\sim-16$ & $\sim-45$ & $+2.5(-1.6)$ & $\sim-4$ & +10.7 \\
300 & $\sim-11$ & $\sim+3$ & $\sim-25$ & $+19.5(+15.8)$ & $\sim+2$ & +3.4 \\
1000 & $\sim-7$ & $\sim+3$ & $\sim-5$ & $+21.4(+2.5)$ & $\sim-2$ & -4 \\
\hline \hline
\end{tabular}




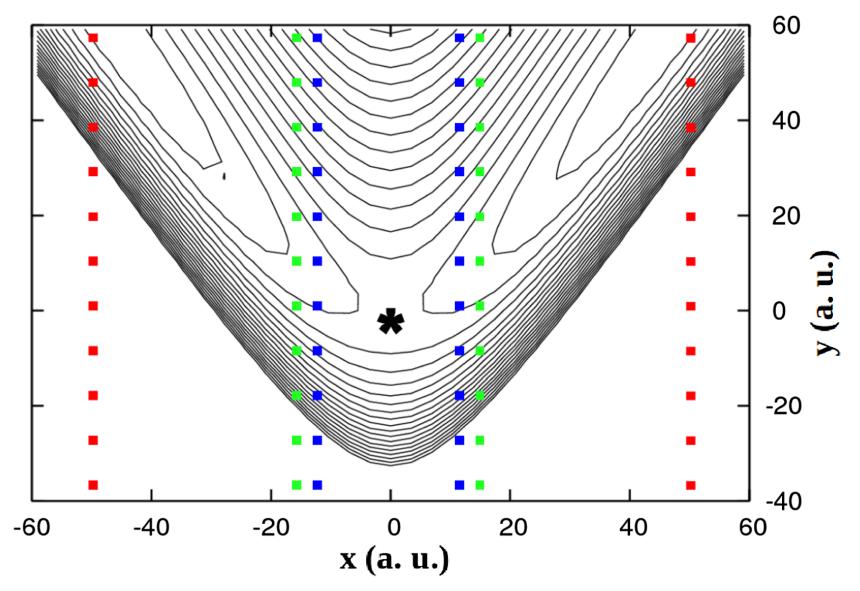

FIG. 5. The LSTH potential energy surface plotted in mass scaled normal mode coordinates for the $\mathrm{H}+\mathrm{H}_{2}$ reaction. The black star represents the location of the transition state. The dotted vertical lines represent three possible positions (blue, green, and red) for the two DSs.

Eq. (15) are calculated again by DVR methods. ${ }^{92}$ For these two dimensional cases, the DSs are assumed to be straight lines, and we employ mass scaled normal mode coordinates ( $\mathrm{x}$ and $y$ ). Initially, we choose scaled normal mode coordinates ( $x$ and $y$ ). Initially, we choose the DSs to be "vertical" surfaces defined by the equation $\mathrm{x}=\mathrm{x}_{1}$ and $\mathrm{x}=\mathrm{x}_{2}$, where $\mathrm{x}$ is the asymmetric stretch mass-scaled normal mode at the transition state, as depicted in Fig. 5. The results obtained with this setup are reported in Table $\mathrm{V}$.

If compared with the one dimensional cases, the results in Table $\mathrm{V}$ are less accurate and a stronger dependence on the DS position can be observed. Nevertheless, the error is still limited to 60 in the worst case scenario.

We now turn to the collinear $\mathrm{D}+\mathrm{H}_{2}$ reaction, where the potential in the mass scaled normal mode coordinates is asymmetric. We set the two DSs in the same fashion as done for the $\mathrm{H}+\mathrm{H}_{2}$, i.e., along the $\mathrm{y}$-coordinate and specularly with respect to the saddle point. We find that the accuracy of rate estimates is sensitive with respect to the position of the two DSs, as reported in Table VI. The same range of precision is found even if we try to set the two DSs in an asymmetric fashion, following the idea we applied to the one-dimensional asymmetric Eckart barrier.

At this point, we observe that the idea of the stationary phase approximation of the flux-flux correlation function

TABLE V. Percentage error $\left[\%\right.$ Err $\left.=100 \times\left(k^{\text {approx }}-k^{\text {exact }}\right) / k^{\text {exact }}\right]$ of the new approach [Eq. (15)]. The rate constants at four temperatures $(150 \mathrm{~K}$, $300 \mathrm{~K}, 600 \mathrm{~K}$, and $1000 \mathrm{~K}$ ) are reported as a function of the position of the right DS. These results are obtained with the DSs placed as in Fig. 5, i.e., vertical and symmetric with respect to the saddle point position.

\begin{tabular}{|c|c|c|c|c|c|c|c|}
\hline \multicolumn{2}{|c|}{$150 \mathrm{~K}$} & \multicolumn{2}{|c|}{$300 \mathrm{~K}$} & \multicolumn{2}{|c|}{$600 \mathrm{~K}$} & \multicolumn{2}{|c|}{$1000 \mathrm{~K}$} \\
\hline $\mathrm{x}_{2}$ & $\%$ Err & $x_{2}$ & $\%$ Err & $\mathrm{x}_{2}$ & $\%$ Err & $\mathrm{x}_{2}$ & $\%$ Err \\
\hline 36 & -20.7 & 16 & +33.0 & 9 & +47.3 & 9 & +19.6 \\
\hline 40 & -18.0 & 18 & +2.1 & 12 & -3.2 & 12 & -21.7 \\
\hline 44 & -10.3 & 20 & -8.5 & 15 & +37.1 & 18 & -8.3 \\
\hline 48 & -5.4 & 24 & -23.1 & 21 & -23.3 & 21 & -36.9 \\
\hline 52 & -0.3 & 28 & -31.2 & 24 & -43.4 & 24 & -54.3 \\
\hline 56 & +4.4 & 30 & -30.4 & 27 & -49.5 & 27 & -38.8 \\
\hline
\end{tabular}

TABLE VI. Worst and best percentage error $\left(\%\right.$ Err $=100 \times\left(k^{\text {approx }}\right.$ $\left.\left.-k^{\text {exact }}\right) / k^{\text {exact }}\right)$ of the approach [Eq. (15)] for the $\mathrm{D}+\mathrm{H}_{2}$ collinear reaction at three different temperatures. These results are obtained with the DS positions as indicated in Fig. 5.

\begin{tabular}{cc}
\hline \hline $\mathrm{T}(\mathrm{K})$ & $\% \operatorname{Err}(\max ; \min )$ \\
\hline 150 & $-34 ;-4.1$ \\
200 & $-59 ;-23$ \\
600 & $-68 ;-13$ \\
\hline \hline
\end{tabular}

integral is based on a fast and a slow part of the time integration. We also consider that the reactive coordinate usually changes faster than the non-reactive ones, and we conclude that the location of the DSs perpendicular to the minimum (classical) energy path (MEP) better suits the rate constant approach presented above. Thus, if we place the DSs as shown in Fig. 6, we obtain the results reported in Table VII. In this way, rate constants are always within $10 \%$ of the exact ones and are almost independent from the position of the DSs. We find a similar accuracy for the isotopic $\mathrm{D}+\mathrm{H}_{2}$ reaction, where the results are in excellent agreement with the exact value of the rate constant, even for symmetric DS locations (see Table VIII). These multidimensional calculations show that the position of the DSs is close to each other at high temperatures and then they become far apart as the temperature is lowered. The explanation for this reasonable behavior is provided by reporting in Fig. 6 the turning points of the Semiclassical Instanton (SCI) paths. The SCI trajectories are imaginary time classical trajectories that represent the analytical continuation in imaginary time of the real time classical trajectories. Pictorially, one can imagine a classical trajectory which represents the time evolution of the reactants approaching the barrier. When the trajectory energy

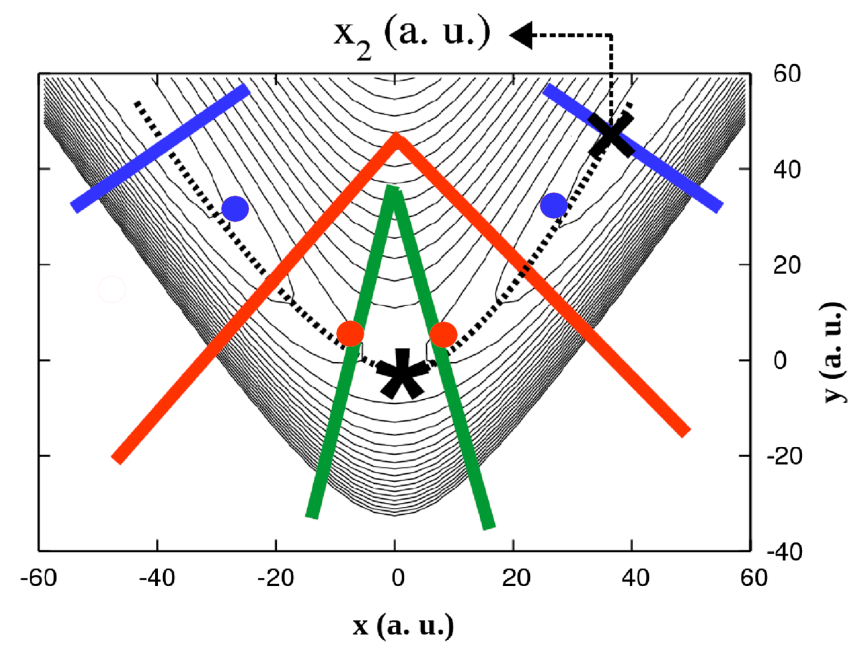

FIG. 6. The DS location for the LSTH potential energy surface plotted in the mass scaled normal mode coordinates for the $\mathrm{H}+\mathrm{H}_{2}$ system. The black star represents the position of the transition state. The potential is symmetric with respect to the transition state. The colored lines represent the three possible positions [blue $(150 \mathrm{~K})$, red $(300 \mathrm{~K})$, and green $(600 \mathrm{~K})$ ] for the two DSs at different temperatures but always perpendicular to the MEP, which is represented by the black dashed curve. The blue and the red dots are the positions of the turning points of the Semiclassical Instanton (SCI) trajectory, respectively, at $150 \mathrm{~K}$ (blue) and $300 \mathrm{~K}$ (red). ${ }^{60}$ The black cross indicates that the position of the DS is provided by the value of the abscissa along the MEP. 
TABLE VII. Percentage error $\left(\%\right.$ Err $\left.=100 \times\left(k^{\text {approx }}-k^{\text {exact }}\right) / k^{\text {exact }}\right)$ of the rate constant calculated with Eq. (15) for the collinear $\mathrm{H}+\mathrm{H}_{2}$ reaction at four temperatures $(150 \mathrm{~K}, 300 \mathrm{~K}, 600 \mathrm{~K}$, and $1000 \mathrm{~K})$. The results are reported as a function of the $\mathrm{x}$ coordinate intersection point between the right DS and the MEP. The DSs are always perpendicular to the MEP and symmetric with respect to the $\mathrm{x}=0$ axis.

\begin{tabular}{|c|c|c|c|c|c|c|c|}
\hline \multicolumn{2}{|c|}{$150 \mathrm{~K}$} & \multicolumn{2}{|c|}{$300 \mathrm{~K}$} & \multicolumn{2}{|c|}{$600 \mathrm{~K}$} & \multicolumn{2}{|c|}{$1000 \mathrm{~K}$} \\
\hline$x_{2}$ & $\%$ Err & $\mathrm{x}_{2}$ & $\%$ Err & $x_{2}$ & $\%$ Err & $x_{2}$ & $\%$ Err \\
\hline 35 & -0.9 & 20 & -4.9 & 20 & -1.5 & 9 & +4.1 \\
\hline 40 & +0.4 & 22 & -9.3 & 22 & -2.4 & 12 & +0.6 \\
\hline 45 & +1.8 & 24 & -10.3 & 25 & -7.7 & 15 & +7.7 \\
\hline 50 & +3.4 & 26 & -10.0 & 30 & -7.0 & 18 & +2.2 \\
\hline 55 & +4.0 & 28 & -9.5 & 32 & -4.1 & 21 & +7.4 \\
\hline 60 & +6.1 & 30 & -9.2 & 35 & -3.4 & 24 & -4.2 \\
\hline
\end{tabular}

is lower than the barrier height, instead of inverting the motion, the trajectory is continued by the instanton periodic trajectory oscillating from one turning point to the other, before continuing as a real time trajectory on the other side of the barrier into the products' valley. The instanton trajectories are the representative of a pure tunneling process and they can be obtained as the periodic trajectories on the inverted potential. ${ }^{27}$ One of us has calculated these periodic trajectories at several temperatures also for the collinear $\mathrm{H}+\mathrm{H}_{2}$ reaction and compared them with a classical TST approach. ${ }^{61}$ The turning points of the instanton periodic trajectories are reported in Fig. 6 as colored dots. As expected from the idea reported in Fig. 1, and according to the derivation that generates Eq. (15), a certain amount of real-time dynamics is calculated from the DSs to the instanton turning points. The distances along the MEP between the DSs and the same colored dot in Fig. 6 allow us to estimate the amount of real-time dynamics that the model foresees. Clearly already at $\mathrm{T}=600 \mathrm{~K}$, the instanton trajectories are not present since the rate is determined by real-time quantum dynamics, which includes quantum reflection contributions.

Finally, Table IX compares the performance of the present approach to other approximate methods that have been applied to the $\mathrm{H}+\mathrm{H}_{2}$ collinear reaction. For instance, the Quantum Transition State Theory (QTST) from Liao and Pollak ${ }^{96}$ and the Mixed Quantum Classical Rate Theory (MQCLT) approach $^{97}$ are very precise at high temperature, but less at low temperature. The QI approximation in its two-DS variant is very accurate, provided that a free-particle correction at high temperatures is employed. Our approximation retains a good

TABLE VIII. The same as in Table VII but for the collinear $\mathrm{D}+\mathrm{H}_{2}$ reaction.

\begin{tabular}{|c|c|c|c|c|c|c|c|}
\hline \multicolumn{2}{|c|}{$150 \mathrm{~K}$} & \multicolumn{2}{|c|}{$300 \mathrm{~K}$} & \multicolumn{2}{|c|}{$600 \mathrm{~K}$} & \multicolumn{2}{|c|}{$1000 \mathrm{~K}$} \\
\hline $\mathrm{x}_{2}$ & $\%$ Err & $\mathrm{x}_{2}$ & $\%$ Err & $\mathrm{x}_{2}$ & $\%$ Err & $x_{2}$ & $\%$ Err \\
\hline 52 & -1.8 & 26 & -8.8 & 17.8 & +0.6 & 7.5 & +7.7 \\
\hline 55 & -0.1 & 31.2 & -8.0 & 21.4 & -0.4 & 9 & +1.3 \\
\hline 58.5 & +1.1 & 36.4 & -8.1 & 24.9 & -2.2 & 10.5 & +6.3 \\
\hline 61.5 & +1.6 & 41.6 & -8.6 & 28.5 & -7.9 & 13.5 & +19.2 \\
\hline 64.6 & +1.9 & 46.8 & -9.0 & 33.8 & -5.5 & 16.5 & +8.8 \\
\hline 67.7 & +2.6 & 52 & -8.4 & 42.75 & -6.9 & 18 & +9.2 \\
\hline
\end{tabular}

TABLE IX. Percentage error $\left(\%\right.$ Err $\left.=100 \times\left(k^{\text {approx }}-k^{\text {exact }}\right) / k^{\text {exact }}\right)$ comparison of the thermal rate constant for the $\mathrm{H}+\mathrm{H}_{2}$ collinear reaction (all with LSTH PES) between different approximate approaches. The values for the QI are obtained with two DSs. The percentage error reported for our method is for the worst case scenario we found.

\begin{tabular}{lcccc}
\hline \hline $\mathrm{T}(\mathrm{K})$ & QTST $^{96}$ & MQCLT $^{97}$ & QI $^{69}$ & Equation (15) \\
\hline 150 & $\ldots$ & $\ldots$ & +10 & +6.1 \\
200 & +270 & +270 & -4.2 & -15.3 \\
300 & +91 & +29.5 & +9.8 & -10.3 \\
1000 & +6 & +3.6 & +3 & -10.9 \\
\hline \hline
\end{tabular}

accuracy (even if worse than the QI one) over the whole temperature range, but without any ad hoc correction. In conclusion, the main advantage of the present method is its simplicity and flexibility because it does not need a precise position of the two DSs along the MEP to get accurate results that could be quite cumbersome for complex reactions. On the contrary, other methods require a preliminary optimization of the DS position.

\section{CONCLUSIONS}

In this paper, we have presented a new quantum approximate method for calculating thermal reaction rate constants. The quantum propagator has to be evaluated at both real and imaginary times, and one of the principal challenges was to find a method that allows for a single time evaluation of the propagator, instead of a complete time evolution. This task is difficult considering that the reaction flux widely oscillates between positive and negative values that almost cancel out. Using the formalism of the flux-flux correlation function for the exact quantum rate formulation, we performed a stationary phase approximation to the time integration after extrapolating the rapidly changing part of the integrand. In this way, we have obtained Eq. (15), which is the central result of this paper. Furthermore, the goal was also to get to a formulation able to provide accurate estimates without the need for a preliminary optimization of the DS positions. We have demonstrated that Eq. (15) includes this feature by applying this single time approximation to well-known one- and bi-dimensional systems, both symmetric and asymmetric, where the substantial quantum effects are mainly responsible for the thermal rate constant behavior. We found that the new formula of Eq. (15) is quite accurate over the entire range of temperatures tested, despite the fact that we do not introduce any ad hoc correction. We verified that the method accounts well for the "corner cutting" observed in the collinear hydrogen exchange reaction at low temperatures. Furthermore, for all systems presented, the accuracy of the results is smoothly dependent on the DS locations. This is important when dealing with complex (high dimensional) systems, since it is not known a priori, where the DSs can be more conveniently placed. The high accuracy of the methods holds as long as the DSs are set sufficiently far apart so that the flux-flux correlation function is zero at time zero. This is a necessary condition for applying the stationary phase approximation, as explained in Sec. II A. However, the method presents some limitations. 
For instance, opposite to classical TST and some semiclassical theories, ${ }^{24}$ this approximation is not a strict upper bound to the exact quantum rate, as it is often the case for QTSTs. Also, the better accuracy of the rate calculation with respect to QTST methods is obtained at the cost of performing realtime quantum dynamics. Future developments will include the possibility to avoid the DVR calculation of the propagator and perform the calculation of the real-time part via semiclassical dynamics. ${ }^{101-106}$ This will introduce a further approximation, since the semiclassical propagator is known to be not suitable in the deep tunneling regime. However, it is quite accurate in the calculation of shallow tunneling and quantum reflection contributions to the rate constant. This foreseen implementation will allow us to calculate the thermal rate constant directly from classical trajectories and without any preliminary Monte Carlo or variational search for the best placement of the DSs. We believe that the present approximation will become very useful when real-time quantum effects are determinant and important for the rate calculation in complex reactions.

\section{ACKNOWLEDGMENTS}

Professor W. H. Miller is warmly thanked for mentoring and inspiring discussions and ideas at the early stage of the project. Dr. Riccardo Conte and Dr. Huaqing Li are thanked for useful discussions. We acknowledge the CINECA and the Regione Lombardia award under the LISA initiative (Grant GREENTI) for the availability of high-performance computing resources. We also thank Università degli Studi di Milano for further computational time at CINECA. C.A. thanks the Università degli Studi di Milano for a Ph.D. scholarship.

\section{APPENDIX A: DERIVATION OF THE FLUX AUTOCORRELATION FUNCTION FOR TWO DIVIDING SURFACES: THE PARABOLIC BARRIER AND FREE PARTICLE CASES}

Here we derive the expression of the flux correlation function for the parabolic barrier with two distinct dividing surfaces and that one of Eq. (24) by taking the limit $\omega \rightarrow 0$. We recall the matrix elements of the parabolic barrier propagator

$$
\left\langle x_{1}\left|e^{-i \hat{H} t_{c} / \hbar}\right| x_{2}\right\rangle=\sqrt{\frac{m \omega}{2 \pi i \hbar \sinh \omega t_{c}}} \exp \left\{\frac{i m \omega}{2 \hbar \sinh \omega t_{c}}\left[\left(x_{1}^{2}+x_{2}^{2}\right) \cosh \omega t_{c}-2 x_{1} x_{2}\right]\right\},
$$

where $\hat{H}$ is given by Eq. (B1), $\mathrm{x}_{1}$ and $\mathrm{x}_{2}$ are the dividing surface positions, and $t_{c}=t-i \hbar \beta / 2 .{ }^{3}$ In the following derivation, we will employ the relations

$$
\begin{aligned}
& \sinh \omega t_{c}=\sinh \omega t \cos (u / 2)-i \cosh \omega t \sin (u / 2), \\
& \cosh \omega t_{c}=\cosh \omega t \cos (u / 2)-i \sinh \omega t \sin (u / 2),
\end{aligned}
$$

where $u=\hbar \omega \beta$. We start from the following expression of the flux-flux correlation function:

$$
\begin{aligned}
C_{f f}(t)= & \left(\frac{\hbar}{2 m}\right)^{2}\left(\left\langle x_{2}\left|e^{-i \hat{H} t_{c}^{*} / \hbar}\right| x_{1}\right\rangle \cdot\left\langle x_{1}\left|e^{-i \hat{H} t_{c} / \hbar}\right| x_{2}\right\rangle+\left\langle x_{2}\left|e^{-i \hat{H} t_{c}^{*} / \hbar}\right| x_{1}\right\rangle \cdot\left\langle x_{1}\left|e^{-i \hat{H} t_{c} / \hbar}\right| x_{2}\right\rangle,\right. \\
& \left.\left.-\left\langle x_{2}\left|e^{-i \hat{H} t_{c}^{*} / \hbar}\right| x_{1}\right\rangle^{\prime}\left\langle x_{1}\left|e^{-i \hat{H} t_{c} / \hbar}\right| x_{2}\right\rangle\right\rangle^{\prime}-\left\langle x_{2}\left|e^{-i \hat{H} t_{c}^{*} / \hbar}\right| x_{1}\right\rangle \cdot\left\langle x_{1}\left|e^{-i \hat{H} t_{c} / \hbar}\right| x_{2}\right\rangle\right),
\end{aligned}
$$

Where, for example, $\left\langle\left\langle x_{1}\left|e^{-i \hat{H} t_{c}^{*} / \hbar}\right| x_{2}\right\rangle=\partial\left\langle x_{2}\left|e^{-i \hat{H} t_{c}^{*} / \hbar}\right| x_{1}\right\rangle / \partial x_{1}\right.$. Using Eq. (A1), the members of Eq. (A3) are

$$
\begin{aligned}
& \left\langle x_{1}\left|e^{-i \hat{H} t_{c} / \hbar}\right| x_{2}\right\rangle=\sqrt{\frac{m \omega}{2 \pi i \hbar \sinh \omega t_{c}}}\left[\frac{i m \omega}{\hbar \sinh \omega t_{c}}\left(x_{1} \cosh \omega t_{c}-x_{2}\right)\right] \exp \left(\frac{i m \omega}{2 \hbar \sinh \omega t_{c}}\left[\left(x_{1}^{2}+x_{2}^{2}\right) \cosh \omega t_{c}-2 x_{1} x_{2}\right]\right), \\
& \left\langle x_{1}\left|e^{-i \hat{H} t_{c} / \hbar}\right| x_{2}\right\rangle^{\prime}=\sqrt{\frac{m \omega}{2 \pi i \hbar \sinh \omega t_{c}}}\left[\frac{i m \omega}{\hbar \sinh \omega t_{c}}\left(x_{2} \cosh \omega t_{c}-x_{1}\right)\right] \exp \left(\frac{i m \omega}{2 \hbar \sinh \omega t_{c}}\left[\left(x_{1}^{2}+x_{2}^{2}\right) \cosh \omega t_{c}-2 x_{1} x_{2}\right]\right), \\
& \left\langle x_{1}\left|e^{-i \hat{H} t_{c} / \hbar}\right| x_{2}\right\rangle^{\prime}=\sqrt{\frac{m \omega}{2 \pi i \hbar \sinh \omega t_{c}}} \exp \left\{\frac{i m \omega}{2 \hbar \sinh \omega t_{c}}\left[\left(x_{1}^{2}+x_{2}^{2}\right) \cosh \omega t_{c}-2 x_{1} x_{2}\right]\right\} \\
& \times\left[-\frac{i m \omega}{\hbar \sinh \omega t_{c}}-\frac{m^{2} \omega^{2}}{\hbar^{2}\left(\sinh \omega t_{c}\right)^{2}}\left(x_{2} \cosh \omega t_{c}-x_{1}\right)\left(x_{1} \cosh \omega t_{c}-x_{2}\right)\right], \\
& \left\langle x_{2}\left|e^{-i \hat{H} t_{c}^{*} / \hbar}\right| x_{1}\right\rangle=\sqrt{\frac{m \omega}{-2 \pi i \hbar \sinh \omega t_{c}^{*}}} \exp \left\{-\frac{i m \omega}{2 \hbar \sinh \omega t_{c}^{*}}\left[\left(x_{1}^{2}+x_{2}^{2}\right) \cosh \omega t_{c}^{*}-2 x_{1} x_{2}\right]\right\} \text {, } \\
& \left\langle x_{2}\left|e^{-i \hat{H} t_{c}^{*} / \hbar}\right| x_{1}\right\rangle=\sqrt{\frac{m \omega}{-2 \pi i \hbar \sinh \omega t_{c}^{*}}}\left[-\frac{i m \omega}{\hbar \sinh \omega t_{c}^{*}}\left(x_{2} \cosh \omega t_{c}^{*}-x_{1}\right)\right] \exp \left\{-\frac{i m \omega}{2 \hbar \sinh \omega t_{c}^{*}}\left[\left(x_{1}^{2}+x_{2}^{2}\right) \cosh \omega t_{c}^{*}-2 x_{1} x_{2}\right]\right\}, \\
& \left\langle x_{2}\left|e^{-i \hat{H} t_{c}^{*} / \hbar}\right| x_{1}\right\rangle^{\prime}=\sqrt{\frac{m \omega}{-2 \pi i \hbar \sinh \omega t_{c}^{*}}}\left[-\frac{i m \omega}{\hbar \sinh \omega t_{c}^{*}}\left(x_{1} \cosh \omega t_{c}^{*}-x_{2}\right)\right] \exp \left\{-\frac{i m \omega}{2 \hbar \sinh \omega t_{c}^{*}}\left[\left(x_{1}^{2}+x_{2}^{2}\right) \cosh \omega t_{c}^{*}-2 x_{1} x_{2}\right]\right\},
\end{aligned}
$$




$$
\begin{aligned}
\left\langle x_{2}\left|e^{-i \hat{H} t_{c}^{*} / \hbar}\right| x_{1}\right\rangle^{\prime}= & \sqrt{\frac{m \omega}{-2 \pi i \hbar \sinh \omega t_{c}^{*}}} \exp \left\{\frac{i m \omega}{2 \hbar \sinh \omega t_{c}^{*}}\left[\left(x_{1}^{2}+x_{2}^{2}\right) \cosh \omega t_{c}-2 x_{1} x_{2}\right]\right\} \\
& \times\left[\frac{i m \omega}{\hbar \sinh \omega t_{c}^{*}}-\frac{m^{2} \omega^{2}}{\hbar^{2}\left(\sinh \omega t_{c}^{*}\right)^{2}}\left(x_{1} \cosh \omega t_{c}^{*}-x_{2}\right)\left(x_{2} \cosh \omega t_{c}^{*}-x_{1}\right)\right] .
\end{aligned}
$$

By inserting the expressions (A1) and (A4)-(A10) into Eq. (A3), we obtain

$$
\begin{aligned}
C_{f f}(t)_{p b}= & \left(\frac{\hbar}{2 m}\right)^{2} \frac{m \omega}{2 \pi \hbar\left|\sinh \omega t_{c}\right|} \exp \left\{\frac { i m \omega } { 2 \hbar | \operatorname { s i n h } \omega t _ { c } | ^ { 2 } } \left[\left(x_{1}^{2}+x_{2}^{2}\right)\left(\cosh \omega t_{c} \sinh \omega t_{c}^{*}-\cosh \omega t_{c}^{*} \sinh \omega t_{c}\right)\right.\right. \\
& \left.\left.+2 x_{1} x_{2}\left(\sinh \omega t_{c}-\sinh \omega t_{c}^{*}\right)\right]\right\}\left[\frac{i m \omega}{\hbar \sinh \omega t_{c}^{*}}-\frac{m^{2} \omega^{2}}{\hbar^{2}\left(\sinh \omega t_{c}^{*}\right)^{2}}\left(x_{1} \cosh \omega t_{c}^{*}-x_{2}\right)\left(x_{2} \cosh \omega t_{c}^{*}-x_{1}\right)-\frac{i m \omega}{\hbar \sinh \omega t_{c}}\right. \\
& -\frac{m^{2} \omega^{2}}{\hbar^{2}\left(\sinh \omega t_{c}\right)^{2}}\left(x_{2} \cosh \omega t_{c}-x_{1}\right)\left(x_{1} \cosh \omega t_{c}-x_{2}\right)-\frac{m^{2} \omega^{2}}{\hbar^{2}\left|\sinh \omega t_{c}\right|}\left(x_{1} \cosh \omega t_{c}^{*}-x_{2}\right)\left(x_{2} \cosh \omega t_{c}-x_{1}\right) \\
& \left.-\frac{m^{2} \omega^{2}}{\hbar^{2}\left|\sinh \omega t_{c}\right|}\left(x_{2} \cosh \omega t_{c}^{*}-x_{1}\right)\left(x_{1} \cosh \omega t_{c}-x_{2}\right)\right]
\end{aligned}
$$

and using the relations Eq. (A2), we obtain the flux-flux correlation function for the parabolic barrier

$$
\begin{aligned}
C_{f f}(t)_{p b}= & \frac{k_{B} T}{h} \frac{u / 2}{\sin (u / 2)} \frac{\omega \sin ^{2}(u / 2) \cosh \omega t}{\left(\sinh ^{2} \omega t+\sin ^{2}(u / 2)\right)^{3 / 2}} \exp \left\{\frac{m \omega \sin (u / 2)}{2 \hbar\left(\sinh ^{2} \omega t+\sin ^{2}(u / 2)\right)}\left[2 x_{1} x_{2} \cosh (\omega t)-\left(x_{1}^{2}+x_{2}^{2}\right) \cos (u / 2)\right]\right\} \\
& \times\left\{1+\left(\frac{2 m}{\hbar}\right)^{2} \frac{\hbar}{2 m \omega \cosh \omega t \sin (u / 2)} \frac{\omega^{2} \sinh ^{2} \omega t}{\left(\sinh ^{2} \omega t+\sin ^{2}(u / 2)\right)}\right. \\
& \left.\times\left[\left(x_{1}^{2}+x_{2}^{2}\right) \cosh \omega t \cos (u / 2)-x_{1} x_{2}\left(\cosh ^{2} \omega t+\cos ^{2}(u / 2)\right)\right]\right\}
\end{aligned}
$$

By taking the limit $\omega \rightarrow 0$, one obtains Eq. (24) for the free particle flux autocorrelation function for arbitrary dividing surface positions.

\section{APPENDIX B: AN ANALYTICAL CASE: THE PARABOLIC BARRIER}

To further investigate the features of the proposed method, it is useful to consider the analytically solvable model of the one-dimensional parabolic barrier. The Hamiltonian for this system is

$$
\hat{H}=\frac{\hat{p}^{2}}{2 m}-\frac{1}{2} m \omega^{2} \hat{x}^{2} .
$$

To compute the rate constant with the approximation in Eq. (15), one has to evaluate the expressions (6), (11), and (12). The matrix elements involved in these calculations are

$$
\begin{gathered}
\left\langle x_{1}\left|e^{-\frac{\beta \hat{H}}{2}-\frac{i \hat{H} t}{\hbar}}\right| x_{2}\right\rangle, \\
\left\langle x_{1}\left|\hat{H} e^{-\frac{\beta \hat{H}}{2}-\frac{\hat{H} t}{\hbar}}\right| x_{2}\right\rangle,
\end{gathered}
$$

$$
\left\langle x_{1}\left|\hat{H}^{2} e^{-\frac{\beta \hat{H}}{2}-\frac{i \hat{H} t}{\hbar}}\right| x_{2}\right\rangle .
$$

By defining the complex time $\beta_{c}=\frac{\beta}{2}+\frac{i t}{\hbar}$, the required matrix elements (B2)-(B4) can be conveniently expressed in terms of $\beta_{c}$ as partial derivatives

$$
\begin{gathered}
\left\langle x_{1}\left|e^{-\beta_{c} \hat{H}}\right| x_{2}\right\rangle, \\
\left\langle x_{1}\left|\hat{H} e^{-\beta_{c} \hat{H}}\right| x_{2}\right\rangle=-\frac{\partial}{\partial \beta_{c}}\left\langle x_{1}\left|e^{-\beta_{c} \hat{H}}\right| x_{2}\right\rangle, \\
\left\langle x_{1}\left|\hat{H}^{2} e^{-\beta_{c} \hat{H}}\right| x_{2}\right\rangle=\frac{\partial^{2}}{\partial \beta_{c}^{2}}\left\langle x_{1}\left|e^{-\beta_{c} \hat{H}}\right| x_{2}\right\rangle .
\end{gathered}
$$

In the parabolic barrier case, the explicit expression for the matrix elements of the complex time propagator is known. ${ }^{3}$ Following the definition of $\beta_{c}$,

$$
\left\langle x_{1}\left|e^{-\beta_{c} \hat{H}}\right| x_{2}\right\rangle_{p b}=\sqrt{\frac{m \omega}{2 \pi \hbar \sin \left(\hbar \omega \beta_{c}\right)}} \exp \left\{-\frac{m \omega}{2 \hbar \sin \left(\hbar \omega \beta_{c}\right)}\left[\left(x_{2}^{2}+x_{1}^{2}\right) \cos \left(\hbar \omega \beta_{c}\right)-2 x_{2} x_{1}\right]\right\},
$$

the partial derivatives in Eqs. (B6) and (B7) can be evaluated analytically,

$$
\begin{aligned}
\left\langle x_{1}\left|\hat{H} e^{-\beta_{c} \hat{H}}\right| x_{2}\right\rangle_{p b}= & \frac{\omega}{4 \sin ^{2}\left(\hbar \beta_{c} \omega\right)} \sqrt{\frac{m \omega}{2 \pi \hbar \sin \left(\hbar \beta_{c} \omega\right)}}\left[-2 m \omega\left(x_{2}^{2}+x_{1}^{2}\right)+4 m \omega x_{1} x_{2} \cos \left(\hbar \beta_{c} \omega\right)+\hbar \sin \left(2 \hbar \beta_{c} \omega\right)\right] \\
& \times \exp \left\{-\frac{m \omega}{2 \hbar}\left[\left(x_{2}^{2}+x_{1}^{2}\right) \frac{\cos \left(\hbar \beta_{c} \omega\right)}{\sin \left(\hbar \beta_{c} \omega\right)}-\frac{2 x_{1} x_{2}}{\sin \left(\hbar \beta_{c} \omega\right)}\right]\right\},
\end{aligned}
$$




$$
\begin{aligned}
\left\langle x_{1}\left|\hat{H}^{2} e^{-\beta_{c} \hat{H}}\right| x_{2}\right\rangle_{p b}= & \frac{\omega^{2}}{32 \sin ^{4}\left(\hbar \beta_{c} \omega\right)} \sqrt{\frac{m \omega}{2 \pi \hbar \sin \left(\hbar \beta_{c} \omega\right)}}\left[-8 \cos \left(2 \beta_{c} \hbar \omega\right)\left(\hbar^{2}-2 m^{2} \omega^{2} x_{1}^{2} x_{2}^{2}\right)-\hbar^{2} \cos \left(4 \beta_{c} \hbar \omega\right)\right. \\
& -32 m^{2} \omega^{2} x_{1} x_{2}\left(x_{1}^{2}+x_{2}^{2}\right) \cos \left(\beta_{c} \hbar \omega\right)-24 \hbar m \omega\left(x_{1}^{2}+x_{2}^{2}\right) \sin \left(2 \beta_{c} \hbar \omega\right) \\
& \left.+48 \hbar m \omega x_{1} x_{2} \sin \left(\beta_{c} \hbar \omega\right)+16 \hbar m \omega x_{1} x_{2} \sin \left(3 \beta_{c} \hbar \omega\right)+9 \hbar^{2}+8 m^{2} \omega^{2}\left(x_{1}^{4}+x_{2}^{4}\right)+32 m^{2} \omega^{2} x_{1}^{2} x_{2}^{2}\right] \\
& \times \exp \left\{-\frac{m \omega}{2 \hbar}\left[\left(x_{2}^{2}+x_{1}^{2}\right) \frac{\cos \left(\hbar \beta_{c} \omega\right)}{\sin \left(\hbar \beta_{c} \omega\right)}-\frac{2 x_{1} x_{2}}{\sin \left(\hbar \beta_{c} \omega\right)}\right]\right\} .
\end{aligned}
$$

In addition, the exact expression for the flux-flux correlation function for an arbitrary position of the two DSs has been derived in Eq. (A12) of Appendix A and by inserting it into the rate expression of Eq. (15), we obtain

$$
\begin{aligned}
k(T) Q_{r}(T)_{p b}^{a p p r o x}= & \frac{\hbar \sqrt{\pi}}{\sqrt{R e \Delta H^{2}\left(\beta, t_{s}\right)}} \exp \left\{\frac{m \omega \sin \left(\frac{\hbar \omega \beta}{2}\right)}{\hbar\left[\sinh ^{2}\left(\omega t_{s}\right)+\sin ^{2}\left(\frac{\hbar \omega \beta}{2}\right)\right]}\left[2 x_{1} x_{2} \cosh \left(\omega t_{s}\right)-\left(x_{1}+x_{2}\right)^{2} \cos \left(\frac{\hbar \omega \beta}{2}\right)\right]\right\} \\
& \times\left\{\cosh \left(\omega t_{s}\right) \sin \left(\frac{\hbar \omega \beta}{2}\right)-\frac{2 m \omega \sinh ^{2}\left(t_{s} \omega\right)}{\hbar\left[\sinh ^{2}\left(\omega t_{s}\right)+\sin ^{2}\left(\frac{\hbar \omega \beta}{2}\right)\right]}\left\{x_{1} x_{2}\left[\cosh ^{2}\left(\omega t_{s}\right)+\cos ^{2}\left(\frac{\hbar \omega \beta}{2}\right)\right]\right.\right. \\
& \left.\left.-\left(x_{1}^{2}+x_{2}^{2}\right) \cosh \left(\omega t_{s}\right) \cos \left(\frac{\hbar \omega \beta}{2}\right)\right\}\right\} \frac{\omega^{2}}{4 \pi\left[\sinh ^{2}\left(\omega t_{s}\right)+\sin ^{2}\left(\frac{\hbar \omega \beta}{2}\right)\right]^{\frac{3}{2}}},
\end{aligned}
$$

where $\Delta H^{2}$ can also be analytically evaluated in terms of the partial derivatives (B6) and (B7) of (B8). Its form after writing explicitly the complex time $\beta_{c}$ according to its definition is

$$
\begin{aligned}
\Delta H_{p b}^{2}= & \frac{8 \hbar \omega^{2} \sin ^{4}\left(\frac{\omega \beta \hbar}{2}-i t \omega\right) \sin \left(\frac{\omega \beta \hbar}{2}+i t \omega\right)}{[\cos (\beta \hbar \omega)-\cosh (2 t \omega)]^{4}} \\
& \times\left[-2 m \omega\left(x_{1}^{2}+x_{2}^{2}\right) \cos \left(\frac{\omega \beta \hbar}{2}+i t \omega\right)+3 m \omega x_{1} x_{2}\right. \\
& \left.+m \omega x_{1} x_{2} \cos (\omega \beta \hbar+i 2 t \omega)+\hbar \sin \left(\frac{\omega \beta \hbar}{2}+i t \omega\right)\right] .
\end{aligned}
$$

Formula (B11) requires to evaluate all the quantities at the stationary time $t_{s}$. In this case, the analytical solution of Eq. (6) can be obtained. To accomplish this task, first, we introduce three constants b, c, and d,

$$
\begin{gathered}
b=4 m \omega x_{1} x_{2} \sin \left(\frac{\hbar \beta \omega}{2}\right), \\
c=2+\cos (\hbar \beta \omega), \\
d=\hbar-2 \hbar \cos (\hbar \beta \omega)-4 m \omega\left(x_{2}^{2}+x_{1}^{2}\right) \sin (\hbar \beta \omega) .
\end{gathered}
$$

Then, Eq. (6) becomes

$$
\begin{gathered}
-\frac{\omega \sinh (t \omega)}{4[\cos (\hbar \beta \omega)-\cosh (2 t \omega)]}\{\hbar \cosh (3 t \omega) \\
+b[c+\cosh (2 t \omega)]+d \cosh (t \omega)\}=0 .
\end{gathered}
$$

The solution of Eq. (B16) provides the time $t_{s}$ for the parabolic barrier potential. A first group of solutions, independent from the position of the two DSs $\mathrm{x}_{1}$ and $\mathrm{x}_{2}$, arises from the first factor in Eq. (B16),

$$
\sin \left(\omega t_{s}\right)=0 \Rightarrow t_{s}=0+\frac{k \pi}{\omega}, \quad k \in \mathbb{N} .
$$

From the second factor, after some algebra and by defining the auxiliary variable $z=\cosh (t \omega)$, we obtain a cubic equation in $\mathrm{z}$,

$$
4 \hbar z^{3}+2 b z^{2}+(d-3 \hbar) z-b+b c=0 .
$$

Its unique real solution is

$$
z=-\frac{b}{6 \hbar}+\frac{\sqrt[3]{2}\left[b^{2}-3 \hbar(d-3 \hbar)\right]}{6 f}+\frac{\sqrt[3]{4}}{12 \hbar} f
$$

where the constant $\mathrm{f}$ is defined as

$$
f=\left\{-2 b^{3}+9 b d \hbar+27 b \hbar^{2}-54 b c \hbar^{2}+\sqrt{-4\left[b^{2}-3 \hbar(d-3 \hbar)\right]^{3}+b^{2}\left[2 b^{2}+9 \hbar(-d-3 \hbar+6 c \hbar)\right]^{2}}\right\}^{\frac{1}{3}} .
$$

Eventually the time $\mathrm{t}_{s}$ is equal to

$$
t_{s}=\frac{1}{\omega} \operatorname{arcosh}\left(-\frac{b}{6 \hbar}+\frac{\sqrt[3]{2}\left[b^{2}-3 \hbar(d-3 \hbar)\right]}{6 f}+\frac{\sqrt[3]{4}}{12 \hbar} f\right) .
$$

It is important to point out that to compute Eq. (B23) one has to take into account the limits of applicability for the definition of the rate, i.e., the rate must be always positive, which translates to the following relation between $\beta$ and the frequency $\omega$ :

$$
0<\frac{\hbar \omega \beta}{2}<\pi \quad \Rightarrow \quad 0<\beta<\frac{2 \pi}{\hbar \omega} .
$$


Starting from the exact parabolic barrier rate value obtained by the time integration of the flux-flux correlation function,

$$
k(T) Q_{r}(T)_{p b}^{e x}=\frac{k_{B} T}{h} \frac{\hbar \omega \beta}{2 \sin \left(\frac{\hbar \omega \beta}{2}\right)},
$$

we look at the ratio between the approximated rate of Eq. (B11) and the exact one of Eq. (B22),

$$
\begin{aligned}
\frac{k(T) Q_{r}(T)_{p b}^{a p p r o x}}{k(T) Q_{r}(T)_{p b}^{e x}}= & \frac{\hbar \sqrt{\pi}}{\sqrt{R e \Delta H^{2}\left(\beta, t_{s}\right)}} \exp \left\{\frac{m \omega \sin \left(\frac{\hbar \omega \beta}{2}\right)}{\hbar\left[\sinh ^{2}\left(\omega t_{s}\right)+\sin ^{2}\left(\frac{\hbar \omega \beta}{2}\right)\right]}\left[2 x_{1} x_{2} \cosh \left(\omega t_{s}\right)-\left(x_{1}^{2}+x_{2}^{2}\right) \cos \left(\frac{\hbar \omega \beta}{2}\right)\right]\right\} \\
& \times\left\{\cosh \left(\omega t_{s}\right) \sin \left(\frac{\hbar \omega \beta}{2}\right)-\frac{2 m \omega \sinh ^{2}\left(t_{s} \omega\right)}{\hbar\left[\sinh ^{2}\left(\omega t_{s}\right)+\sin ^{2}\left(\frac{\hbar \omega \beta}{2}\right)\right]}\left\{x_{1} x_{2}\left[\cosh ^{2}\left(\omega t_{s}\right)+\cos ^{2}\left(\frac{\hbar \omega \beta}{2}\right)\right]\right.\right. \\
& \left.\left.-\left(x_{1}^{2}+x_{2}^{2}\right) \cosh \left(\omega t_{s}\right) \cos \left(\frac{\hbar \omega \beta}{2}\right)\right\}\right\} \frac{\omega \sin \left(\frac{\hbar \omega \beta}{2}\right)}{\left[\sinh ^{2}\left(\omega t_{s}\right)+\sin ^{2}\left(\frac{\hbar \omega \beta}{2}\right)\right]^{\frac{3}{2}}} .
\end{aligned}
$$

The evaluation of this ratio and the calculation of the time $t_{s}$ have been carried out analytically by solving Eq. (6).

To test the present approximation, we choose the potential parameters so that the inverted parabola has a (downward) curvature which corresponds to the frequency of the $\mathrm{H}_{2}$ molecule vibration. The mass $\mathrm{m}=918$ a.u. is equal to the reduced mass of $\mathrm{H}_{2}$, and the frequency is $\omega=0.020056988$ a.u. With these parameters, the range of validity of the rate expression according to the condition (B21) is $0<\beta<313.27$ a.u. This means we can estimate rate constants only for temperatures higher than $1007 \mathrm{~K}$, that is, the threshold temperature when studying this model. We have investigated the outcomes

TABLE X. Minimum distance between the two DSs that gives a rate constant estimate within $10 \%$ of the exact result. The two DSs are set symmetrically with respect to the top of the barrier.

\begin{tabular}{ll}
\hline \hline $\mathrm{T}(\mathrm{K})$ & $\Delta \mathrm{x}^{\mathrm{min}}($ a.u. $)$ \\
\hline 1300 & 0.44 \\
1500 & 0.33 \\
2000 & 0.22 \\
2500 & 0.19 \\
\hline \hline
\end{tabular}

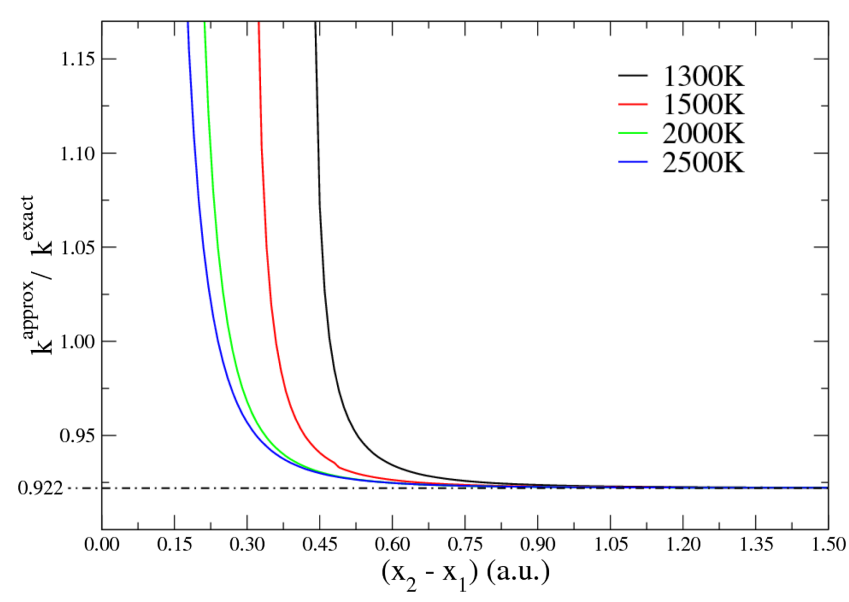

FIG. 7. Ratio of the approximate rate constant value and the exact one for the parabolic barrier as a function of the distance between the two DSs. For all temperatures, the same limit value of precision is reached. of Eq. (B23) from $1300 \mathrm{~K}$ up to $2500 \mathrm{~K}$ as a representative range of temperatures. When the temperature is high, the two DSs have to be set close to each other to find an acceptable value of $\mathrm{t}_{s}$, while decreasing the temperature the distance between the surfaces becomes larger, as summarized in Table X. The approximation that we have introduced is not exact for the parabolic barrier. However, at all temperatures, we have found that increasing the distance between the two DSs, the rate estimates gradually decrease until a limit to the precision of the approximate rate is reached, as depicted in Fig. 7.

${ }^{1}$ W. H. Miller, J. Phys. Chem. A 102, 793 (1998).

${ }^{2}$ W. H. Miller, J. Chem. Phys. 50, 407 (1969).

${ }^{3}$ W. H. Miller, S. D. Schwartz, and J. W. Tromp, J. Chem. Phys. 79, 4889 (1983).

${ }^{4}$ M. S. Small, C. Predescu, and W. H. Miller, Chem. Phys. 322, 151 (2006).

${ }^{5}$ T. J. Park and J. Light, J. Chem. Phys. 88, 4897 (1988).

${ }^{6}$ J. W. Tromp and W. H. Miller, Faraday Discuss. Chem. Soc. 84, 441 (1987).

${ }^{7}$ H. Wang, W. H. Thompson, and W. H. Miller, J. Chem. Phys. 107, 7194 (1997).

${ }^{8}$ P. N. Day and D. G. Truhlar, J. Chem. Phys. 95, 5097 (1991).

${ }^{9}$ W. H. Thompson and W. H. Miller, J. Chem. Phys. 106, 142 (1997).

${ }^{10}$ U. Manthe and F. Matzkies, Chem. Phys. Lett. 282, 442 (1998).

${ }^{11}$ F. Matzkies and U. Manthe, J. Chem. Phys. 108, 4828 (1998).

${ }^{12}$ F. Huarte-Larrañaga and U. Manthe, J. Phys. Chem. A 105, 2522 (2001).

${ }^{13}$ L. Huaqing, J. Poulsen, and G. Nyman, J. Phys. Chem. Lett. 4, 3013 (2013).

${ }^{14}$ H. Li, J. A. Poulsen, and G. Nyman, J. Phys. Chem. A 115, 7338 (2011).

${ }^{15}$ E. Martin-Fierro and E. Pollak, J. Chem. Phys. 125, 164104 (2006).

${ }^{16}$ J. A. Poulsen, H. Li, and G. Nyman, J. Chem. Phys. 131, 024117 (2009).

${ }^{17}$ C. Venkataraman and W. H. Miller, J. Chem. Phys. 126, 094104 (2007).

${ }^{18}$ H. Wang, M. Thoss, and W. H. Miller, J. Chem. Phys. 112, 47 (2000).

${ }^{19}$ H. Wang, M. Thoss, K. L. Sorge, R. Gelabert, X. Giménez, and W. H. Miller, J. Chem. Phys. 114, 2562 (2001).

${ }^{20}$ T. Yamamoto and W. H. Miller, J. Chem. Phys. 120, 3086 (2004).

${ }^{21}$ T. Yamamoto, H. Wang, and W. H. Miller, J. Chem. Phys. 116, 7335 (2002).

${ }^{22}$ H. Eyring, J. Chem. Phys. 3, 107 (1935).

${ }^{23}$ E. Wigner, J. Chem. Phys. 5, 720 (1937).

${ }^{24}$ D. G. Truhlar and B. C. Garrett, Acc. Chem. Res. 13, 440 (1980).

${ }^{25}$ E. Wigner, Trans. Faraday Soc. 34, 29 (1938).

${ }^{26}$ H. Eyring, Trans. Faraday Soc. 34, 41 (1938).

${ }^{27}$ W. H. Miller, J. Chem. Phys. 62, 1899 (1975).

${ }^{28}$ G. A. Voth, D. Chandler, and W. H. Miller, J. Chem. Phys. 91, 7749 (1989).

${ }^{29}$ D. G. Truhlar and B. C. Garrett, J. Phys. Chem. 96, 6515 (1992).

${ }^{30}$ N. F. Hansen and H. C. Andersen, J. Phys. Chem. 100, 1137 (1996).

${ }^{31}$ T. Yamamoto and W. H. Miller, J. Chem. Phys. 122, 044106 (2005). 
${ }^{32}$ S. Mandrà, J. Schrier, and M. Ceotto, J. Phys. Chem. A 118, 6457 (2014).

${ }^{33}$ S. Mandrà, S. Valleau, and M. Ceotto, Int. J. Quantum Chem. 113, 1722 (2013).

${ }^{34}$ M. Topaler and N. Makri, J. Chem. Phys. 101, 7500 (1994).

${ }^{35}$ N. J. Wright and N. Makri, J. Chem. Phys. 119, 1634 (2003).

${ }^{36}$ M. Topaler and N. Makri, Chem. Phys. Lett. 210, 285 (1993).

${ }^{37}$ J. Liu and W. H. Miller, J. Chem. Phys. 127, 114506 (2007).

${ }^{38}$ M. Thoss, H. Wang, and W. H. Miller, J. Chem. Phys. 115, 2991 (2001).

${ }^{39}$ T. F. Miller III and D. E. Manolopoulos, J. Chem. Phys. 122, 184503 (2005).

${ }^{40}$ E. Pollak, J. Chem. Phys. 127, 074505 (2007).

${ }^{41}$ W. H. Miller, Faraday Discuss. 110, 1 (1998).

${ }^{42}$ N. Makri, Annu. Rev. Phys. Chem. 50, 167 (1999).

${ }^{43}$ A. Nakayama and N. Makri, J. Chem. Phys. 119, 8592 (2003).

${ }^{44}$ J. Liu and W. H. Miller, J. Chem. Phys. 126, 234110 (2007).

${ }^{45}$ M. Ceotto, S. Yang, and W. H. Miller, J. Chem. Phys. 122, 044109 (2005).

${ }^{46}$ E. Sim, G. Krilov, and B. Berne, J. Phys. Chem. A 105, 2824 (2001).

${ }^{47}$ S. Bonella, M. Monteferrante, C. Pierleoni, and G. Ciccotti, J. Chem. Phys. 133, 164104 (2010).

${ }^{48}$ Q. Shi and E. Geva, J. Phys. Chem. A 108, 6109 (2004).

${ }^{49}$ I. R. Craig and D. E. Manolopoulos, J. Chem. Phys. 122, 084106 (2005).

${ }^{50}$ E. Pollak and E. Martin-Fierro, J. Chem. Phys. 126, 164107 (2007).

${ }^{51}$ W. Zhu and Y. Zhao, J. Chem. Phys. 129, 184111 (2008).

${ }^{52}$ S. Bonella, D. Montemayor, and D. F. Coker, Proc. Natl. Acad. Sci. U. S. A. 102, 6715 (2005).

${ }^{53}$ N. Markovic and J. A. Poulsen, J. Phys. Chem. A 112, 1701 (2008).

${ }^{54}$ J. A. Poulsen, G. Nyman, and P. J. Rossky, J. Chem. Phys. 119, 12179 (2003).

${ }^{55}$ R. Conte and E. Pollak, Phys. Rev. E 81, 036704 (2010).

${ }^{56}$ R. Conte and E. Pollak, J. Chem. Phys. 136, 094101 (2012).

${ }^{57}$ R. Conte, A. Aspuru-Guzik, and M. Ceotto, J. Phys. Chem. Lett. 4, 3407 (2013).

${ }^{58}$ D. Tamascelli, F. S. Dambrosio, R. Conte, and M. Ceotto, J. Chem. Phys. 140, 174109 (2014).

${ }^{59}$ R. Hernandez and W. H. Miller, Chem. Phys. Lett. 214, 129 (1993).

${ }^{60}$ T. L. Nguyen, J. F. Stanton, and J. R. Barker, J. Phys. Chem. A 115, 5118 (2011).

${ }^{61}$ S. M. Greene, X. Shan, and D. C. Clary, J. Phys. Chem. A 119, 12015 (2015).

${ }^{62}$ C. Aieta, F. Gabas, and M. Ceotto, J. Phys. Chem. A 120, 4853 (2016).

${ }^{63}$ M. Ceotto, Mol. Phys. 110, 547 (2012).

${ }^{64}$ M. Kryvohuz, J. Chem. Phys. 134, 114103 (2011).

${ }^{65}$ I. R. Craig and D. E. Manolopoulos, J. Chem. Phys. 121, 3368 (2004).

${ }^{66}$ J. O. Richardson and S. C. Althorpe, J. Chem. Phys. 131, 214106 (2009).

${ }^{67}$ T. J. Hele and S. C. Althorpe, J. Chem. Phys. 138, 084108 (2013).

${ }^{68}$ S. C. Althorpe and T. J. Hele, J. Chem. Phys. 139, 084115 (2013).

${ }^{69}$ J. Cao and G. A. Voth, J. Chem. Phys. 100, 5093 (1994).

${ }^{70}$ E. Geva, Q. Shi, and G. A. Voth, J. Chem. Phys. 115, 9209 (2001).

${ }^{71}$ Q. Shi and E. Geva, J. Chem. Phys. 119, 9030 (2003).

${ }^{72}$ W. H. Miller, Y. Zhao, M. Ceotto, and S. Yang, J. Chem. Phys. 119, 1329 (2003).

${ }^{73}$ M. Ceotto and W. H. Miller, J. Chem. Phys. 120, 6356 (2004).
${ }^{74}$ Y. Zhao, T. Yamamoto, and W. H. Miller, J. Chem. Phys. 120, 3100 (2004).

${ }^{75}$ M. Buchowiecki and J. Vaníček, J. Chem. Phys. 132, 194106 (2010).

${ }^{76} \mathrm{~W}$. Wang and Y. Zhao, J. Chem. Phys. 132, 064502 (2010).

${ }^{77}$ W. Wang and Y. Zhao, J. Chem. Phys. 130, 114708 (2009).

${ }^{78}$ J. Vaníček, W. H. Miller, J. F. Castillo, and F. J. Aoiz, J. Chem. Phys. 123, 054108 (2005).

${ }^{79}$ J. Vaníček and W. H. Miller, J. Chem. Phys. 127, 114309 (2007).

${ }^{80}$ J. Huang, M. Buchowiecki, T. Nagy, J. Vaníček, and M. Meuwly, Phys. Chem. Chem. Phys. 16, 204 (2014).

${ }^{81}$ T. Zimmermann and J. Vaníček, J. Chem. Phys. 131, 024111 (2009).

${ }^{82}$ M. Buchowiecki and J. Vaníček, Chem. Phys. Lett. 588, 11 (2013).

${ }^{83}$ K. Karandashev and J. Vaníček, J. Chem. Phys. 143, 194104 (2015).

${ }^{84}$ R. Conte, P. L. Houston, and J. M. Bowman, J. Phys. Chem. A 117, 14028 (2013).

${ }^{85}$ R. Conte, B. Fu, E. Kamarchik, and J. M. Bowman, J. Chem. Phys. 139, 044104 (2013).

${ }^{86}$ R. Conte, P. L. Houston, and J. M. Bowman, J. Phys. Chem. A 118, 7742 (2014).

${ }^{87}$ R. Conte, P. L. Houston, and J. M. Bowman, J. Chem. Phys. 140, 151101 (2014).

${ }^{88}$ P. L. Houston, R. Conte, and J. M. Bowman, J. Phys. Chem. A 118, 7758 (2014).

${ }^{89}$ R. Conte, C. Qu, and J. M. Bowman, J. Chem. Theory Comput. 11, 1631 (2015).

${ }^{90}$ C. Qu, R. Conte, P. L. Houston, and J. M. Bowman, Phys. Chem. Chem. Phys. 17, 8172 (2015).

${ }^{91}$ Z. Homayoon, R. Conte, C. Qu, and J. M. Bowman, J. Chem. Phys. 143, 084302 (2015).

${ }^{92}$ R. Conte, P. L. Houston, and J. M. Bowman, J. Phys. Chem. A 119, 12304 (2015).

${ }^{93}$ P. L. Houston, R. Conte, and J. M. Bowman, J. Phys. Chem. A 119, 4695 (2015).

${ }^{94}$ P. L. Houston, R. Conte, and J. M. Bowman, J. Phys. Chem. A 120, 5103 (2016).

${ }^{95}$ D. T. Colbert and W. H. Miller, J. Chem. Phys. 96, 1982 (1992).

${ }^{96} \mathrm{M}$. Baer, Theory of Chemical Reaction Dynamics (CRC Press, Inc., Boca Raton, FL, 1985), Vol. 3.

${ }^{97}$ P. Siegbahn and B. Liu, J. Chem. Phys. 68, 2457 (1978).

${ }^{98}$ D. G. Truhlar and C. J. Horowitz, J. Chem. Phys. 68, 2466 (1978).

${ }^{99}$ J.-L. Liao and E. Pollak, J. Phys. Chem. A 104, 1799 (2000).

${ }^{100}$ Y. Zheng and E. Pollak, J. Chem. Phys. 114, 9741 (2001).

${ }^{101}$ W. H. Miller, Proc. Natl. Acad. Sci. U.S.A. 102, 6660 (2005).

${ }^{102}$ K. G. Kay, Chem. Phys. 322, 3 (2006).

${ }^{103}$ Y. Zhuang, M. R. Siebert, W. L. Hase, K. G. Kay, and M. Ceotto, J. Chem. Theory Comput. 9, 54 (2013).

${ }^{104}$ M. Ceotto, Y. Zhuang, and W. L. Hase, J. Chem. Phys. 138, 054116 (2013).

${ }^{105}$ M. Ceotto, S. Valleau, G. F. Tantardini, and A. Aspuru-Guzik, J. Chem. Phys. 134, 234103 (2011).

${ }^{106}$ F. Gabas, R. Conte, and M. Ceotto, "On-the-fly ab initio semiclassical calculation of glycine vibrational spectrum," J. Chem. Theory Comput. (published online). 